\title{
Exclusión social y género: un análisis de la realidad contemporánea ${ }^{1}$
}

\section{Paola Damonti}

Dpto. de Trabajo Social, Universidad Pública de Navarra

<paola.damonti@unavarra.es>

Artikulu honetan garatzen dugu genero-ikuspegia kontuan hartzen duen gizarte-bazterketaren azterketa bat. Batetik, azaltzen dugu gaur egungo genero-desberdintasunen argazki bat; eta bestetik, azterketa estatiko horrek lagun hartzen du joandako zazpi urteotan gertatutako transformazioen inguruko azterlan dinamiko bat. Hori dela-eta, jo genuen Gizarteratze eta Premia Sozialen inguruko Foessa Fundazioaren Inkesten 2007, 2009 eta 2013. urteko analisietara. Iturri horien bitartez aurkezten dira bai indibiduo bai etxebizitzaren inguruko datuak, dela modu agregatuan, dela gizarte-bazterketaren dimentsio desberdinen arabera desberdinduz, eta biztanleria osoaren inguruko datuak edota bereziki kaltebera diren kolektiboen arabera. Paraleloki, pobreziaren feminitatearen nozioa kontuan hartuz berresteko erabiltzen ditugu eskuragarri dauden datu enpirikoak, edo aitzitik, gezurtatuz kontzeptua azaltzean erabilitako zenbait adiera.
En este artículo desarrollamos un análisis de la exclusión social con perspectiva de género: por un lado, presentamos una fotografía de las desigualdades de género existentes en la actualidad; por otro, a este análisis estático se le acompaña de un estudio dinámico de las transformaciones que han tenido lugar en los últimos siete años. Para ello, recurrimos a las Encuestas de la Fundación Foessa sobre Integración Social y Necesidades Sociales de 2007, 2009 y 2013. A partir de estas fuentes, presentamos datos tanto a nivel tanto individual como de hogar, tanto agregados como diferenciando las distintas dimensiones de la exclusión, y tanto relativos al conjunto de la población como focalizados en colectivos especialmente vulnerables. Paralelamente, considerando la importancia de la noción de feminización de la pobreza, utilizamos los datos empíricos disponibles para confirmar 0 , al contrario, desmentir las distintas acepciones con las cuales se ha utilizado este concepto.

\section{Palabras Clave:}

Exclusión social, género, desigualdades de género, crisis económica, análisis cuantitativo. 


\section{Introducción}

En el presente artículo desarrollaremos un análisis de la exclusión social con perspectiva de género: por un lado, presentaremos una fotografía de las desigualdades de género existentes en la actualidad en lo relativo a la integración/exclusión social; por otro, a este análisis estático le acompañará un estudio dinámico de las transformaciones que han tenido lugar en los últimos siete años, en concomitancia con una época de profunda crisis económica.

Empezaremos este trabajo analizando cómo el género influye en los procesos de exclusión social, a nivel tanto individual como de hogar. Esta diferenciación es necesaria, ya que, como veremos, un análisis a nivel de individuos puede llevarnos a una infraestimación de las desigualdades de género existentes. En segundo lugar, afinaremos el análisis y focalizaremos la mirada en las dimensiones e indicadores que conforman los procesos de exclusión social antes nombrados. De esta manera, podremos mejorar nuestra comprensión de cómo el género incide tanto en los factores que desencadenan tales procesos como en su intensidad y en los mecanismos que se utilizan para salir de ellos (Pérez y Laparra, 2007). Después, volveremos a centrar la mirada en la dimensión económica, observando cómo el sexo de la persona (no) empleada tiene consecuencias de cara a los procesos de exclusión. En cuarto lugar, una vez realizado este análisis para el conjunto de la población, centraremos nuestra mirada en colectivos especialmente vulnerables (personas de origen extranjero, jóvenes y hogares monoparentales). Paralelamente, considerando que la noción de feminización de la pobreza ha ocupado un lugar casi hegemónico en las investigaciones que estudian la pobreza y la exclusión desde una perspectiva de género, realizaremos también un análisis crítico de este concepto. En concreto, utilizaremos los datos empíricos disponibles para confirmar o, al contrario, desmentir las distintas acepciones con las cuales se utiliza este concepto.

Antes de adentrarnos en el análisis, sin embargo, es necesario definir los conceptos a los que recurriremos para desarrollarlo, y más en concreto, las nociones de exclusión social, por un lado, y de feminización de la pobreza, por otro.

\section{Aproximación teórica}

\subsection{La noción de exclusión social}

El concepto de exclusión social se empezó a utilizar en Francia en los años setenta, y entró a formar parte del lenguaje de la Unión Europea a partir del final de la década siguiente, como sustituto de la idea de pobreza, objeto de críticas generalizadas por su orientación exclusivamente economicista (Subirats, 2004; Laparra et al., 2007). Más de dos décadas después, su utilización se ha generalizado. Su significado, sin embargo, permanece relativamente confuso: las instancias que lo utilizan son tantas y tan distintas que es virtualmente imposible llegar a una definición aceptada por todas, sobre todo si consideramos que, al tratarse un concepto social y políticamente sensible, una definición absoluta simplemente no existe (Paugam, 2000). Por ello es tan relevante el acuerdo al que han llegado varios grupos de investigación del Estado, al definir la exclusión como "un proceso social de pérdida de integración que incluye no sólo la falta de ingresos y el alejamiento del mercado de trabajo, sino también un descenso en la participación social y, por tanto, una pérdida de derechos sociales" (Laparra et al., 2007: 25). Esta noción de exclusión implica la existencia de tres aspectos fundamentales en las situaciones de dificultad: su origen estructural, su carácter multidimensional y su naturaleza procesual.

En primer lugar, nos encontramos frente a un fenómeno de origen estructural, cuya comprensión, por lo tanto, requiere un análisis de las tres principales estructuras de integración: mercado laboral, redes sociales y familiares, y Estado del bienestar. En lo que se refiere al mercado laboral, hay que destacar, por un lado, el riesgo de descualificación, resultante de un contexto de cambios tecnológicos acelerados; y por otro, el empeoramiento de la calidad del trabajo, en forma de aumento del trabajo precario, discontinuo y sumergido. Este aumento de la precariedad en el mercado laboral se ve agravado por una reestructuración del Estado del bienestar que conlleva una disminución de su capacidad protectora (Rodríguez, 2004). Finalmente, al panorama hasta aquí presentado hay que añadir los efectos de las trasformaciones acaecidas en el seno de la familia (disminución del tamaño de los hogares y aumento de los monoparentales, unidos al envejecimiento de la población), que han mermado su capacidad integradora.

En segundo lugar, la exclusión es un concepto multidimensional, que "no se puede explicar con arreglo a una única causa o factor, sino que precisamente se define por una acumulación de factores o déficits que se interrelacionan y retroalimentan entre sí" (Subirats, 2004: 19). Para identificarla, por lo tanto, es necesario observar varias dimensiones conjuntamente, tal y como se especifica en el Cuadro 1.

Finalmente, la exclusión es un fenómeno de carácter procesual, dinámico y en constante expansión, que no debe ser entendido como un estado, sino como un "proceso de alejamiento de algunos individuos respecto al centro de la sociedad" (Subirats, 2004; Laparra et al., 2007: 35).

\subsection{De la feminización de la pobreza a un análisis de la exclusión con perspectiva de género}

Con respecto a la dimensión de género, cabe destacar que, durante largo tiempo, fue ignorada por las investigaciones que se ocupaban de la pobreza y la exclusión social. Esto significa que las diferencias 


\begin{tabular}{|c|c|c|}
\hline \multicolumn{3}{|c|}{ Cuadro 1. Dimensiones de la exclusión social } \\
\hline Ejes & Dimensiones & Aspectos \\
\hline \multirow{2}{*}{ Económico } & Participación en la producción & - Exclusión de la relación salarial normalizada \\
\hline & Participación en el consumo & $\begin{array}{l}\text { - Pobreza económica } \\
\text { - Privación }\end{array}$ \\
\hline \multirow{2}{*}{ Político } & Ciudadanía política & $\begin{array}{l}\text { - Acceso efectivo a los derechos políticos } \\
\text { - Abstencionismo y pasividad política }\end{array}$ \\
\hline & Ciudadanía social & $\begin{array}{l}\text { - Acceso limitado a los sistemas de protección social: sanidad, vivienda } \\
\text { y educación }\end{array}$ \\
\hline \multirow{2}{*}{ Social (relacional) } & Ausencia de lazos sociales & - Aislamiento social, falta de apoyos sociales \\
\hline & Relaciones sociales perversas & $\begin{array}{l}\text { - Integración en redes sociales ‘desviadas' } \\
\text { - Conflictividad social (conductas anómicas) y familiar (violencia } \\
\text { doméstica) }\end{array}$ \\
\hline
\end{tabular}

Fuente: Laparra, Zugasti y García Lautre (2014).

entre hombres y mujeres -en términos tanto de incidencia de los fenómenos estudiados como de sus causas y consecuencias- no se medían, ni mucho analizaban en profundidad. Esta 'ceguera' frente al género fue puesta en tela de juicio por primera vez por las investigadoras que formularon el concepto de feminización de la pobreza, acuñado en 1978 por Diana Pearce (Aguilar, 2011) y que, sin embargo, alcanzó notoriedad sólo años más tarde, con la publicación, en 1984, de la obra de Hilda Scott Working Your Way to the Bottom. The Feminization of Poverty (Fernández Viguera, 1998).

Desde entonces, la noción de feminización de la pobreza ha logrado una difusión cada vez mayor (Brunet, Valls y Belzunegui, 2008), aunque con referencia a un abanico de significados muy amplios y diversos (Fernández Viguera, 1998; Aguilar, 2011), lo cual, como veremos, la convierte en un concepto históricamente muy importante pero actualmente poco preciso. En concreto, este concepto se ha usado para reflejar:

- La sobrerrepresentación de las mujeres en la franja de pobreza (Mateo Pérez, 2000; Herr et al., 2003, cit. en Kim y Choi, 2013; Brady y Kall, 2008; Aguilar, 2011).

- El aumento progresivo de la presencia de mujeres entre las personas pobres (Fernández Viguera, 1992 y 1998; Cabrera, 2004; Brunet, Valls y Belzunegui, 2008; Tortosa, 2009; Aguilar, 2011; Kim y Choi, 2013).

- El incremento de la pobreza en hogares encabezados por mujeres (Aguilar, 2011).

- El aumento de la visibilidad de la pobreza de las mujeres (Fernández Viguera, 1992 y 1998; Aguilar, 2011).

- El hecho de las mujeres se empobrecen por razones y procesos específicos y condicionados por el género (Fernández Viguera, 1998; Mateo Pérez, 2000; Aguilar, 2011).
En resumen, podemos afirmar que se trata de un concepto atravesado por luces y sombras. Por un lado, es en gran parte gracias a las investigadoras que se han ocupado de este tema que la perspectiva de género se ha incluido en los análisis sobre pobreza. Por otra, el innegable valor de estas aportaciones no puede hacernos olvidar que estamos frente a un concepto "tan llevado y traído como confuso, difícil y equívoco" (De Elejabeitia, 1996: 172). Tal confusión está en directa relación con la polisemia del concepto, que, como se ha visto, se utiliza para nombrar procesos y realidades profundamente diferentes entre sí (Medeiros y Costa, 2008), y a veces, incluso, llega a utilizarse sin ni siquiera aclarar el significado específico que se le atribuye (Aguilar, 2011). Todos estos elementos nos llevan a poner en tela de juicio la oportunidad de seguir utilizándolo, sobre todo cuando no se acompaña de una clara definición del significado que se le atribuye. Conscientes de la importancia que dicho concepto tiene en términos históricos, y con ánimo de aportar algo de claridad a este panorama confuso, a lo largo de este trabajo utilizaremos, sin embargo, los datos disponibles para confirmar 0 desmentir cada una de las acepciones con las que se utiliza la noción de feminización de la pobreza.

\section{Metodología}

Para conseguir los objetivos que nos hemos propuesto, hemos utilizado una metodología cuantitativa. En concreto, hemos realizado un análisis de los microdatos de las Encuestas de la Fundación Foessa sobre Integración Social y Necesidades Sociales, en sus tres ediciones (2007, 2009 y 2013). Estas encuestas, que han sido diseñadas específicamente para estudiar los fenómenos que nos ocupan, resultan especialmente adecuadas para nuestros objetivos, por varias razones:

- En primer lugar, porque el análisis de los procesos de exclusión que realizan es muy detallado 
y recurre a 35 indicadores, a su vez relacionados con 8 dimensiones de la exclusión. Constituyen, en suma, la fuente de datos más completa a este respecto a nivel estatal.

- En segundo lugar, porque el muestreo se ha realizado de manera que garantiza una sobrerrepresentación de hogares en situación de exclusión (luego corregida por medio de una cuidadosa ponderación) ${ }^{2}$. Esto resulta de fundamental interés para el análisis, ya que permite obtener un número suficiente de hogares con indicios de exclusión social para realizar estudios, a la vez, detallados y precisos.

- Finalmente, porque se trata de encuestas que ya tienen cierto recorrido: de hecho, hasta la fecha se han realizado tres ediciones, tanto antes como después de la crisis económica. Esto nos ofrece grandes posibilidades en términos comparativos y nos permite presentar no solamente datos estáticos, sino también análisis de tendencias.

Con respecto a la ponderación de los 35 indicadores que se han utilizado para medir la exclusión, cabe destacar que no todos tienen el mismo peso. Al contrario, en cada dimensión (participación en la producción, participación en el producto social, derechos políticos, conflicto social y aislamiento social) se han sumado los inversos de las frecuencias de cada indicador (1/frecuencia) y el resultado se ha dividido por el número total de indicadores de dicha dimensión. El valor obtenido representa el peso de cada indicador. Después, estas cifras se han normalizado para que la suma tanto de los indicadores de cada dimensión como del conjunto de indicadores sea 1. La suma ponderada de los 35 indicadores representa el Índice Sintético de Exclusión Social (ISES), que tiene valor mínimo igual a o y media igual a 1 (no hay límite máximo). A partir de aquí, se han creado cuatro categorías:

1. Integración: $\mathbf{i}=0$.

2. Exclusión precaria: $0<\mathrm{i}<2$.

3. Exclusión compensada: $2<\mathrm{i}<4$.

4. Exclusión severa: $\mathrm{i}>4$.

Aunque este proceso se realizó en cada edición de la encuesta, en 2013 se decidió que, a fines comparativos, lo más oportuno era aplicar a las bases de 2009 y 2013 los pesos de 2007. Esto implica que el ISES medio será igual a 1 solamente en 2007 , pudiendo ser en 2009 y 2013 más elevado o más reducido (es, obviamente, más elevado) ${ }^{3}$.

Una vez aclaradas estas cuestiones de carácter metodológico, podemos adentrarnos en el análisis.

${ }^{2}$ Para un análisis más detallado de las cuestiones metodológicas relativas al diseño e implementación de la encuesta, se remite al trabajo de Trujillo Carmona (2014).

3 Para un análisis más detallado de las cuestiones metodológicas relativas a la operacionalización de la noción de exclusión, se remite al trabajo de Laparra, Zugasti y García Lautre (2014).

\section{Exclusión social: se reducen las diferencias sobre base individual}

Empezamos nuestro estudio realizando un análisis sobre base individual. Descubrimos así que, entre 2007 y 2013, las diferencias en el nivel de integración de hombres y mujeres han ido reduciéndose hasta prácticamente desaparecer. Si hace siete años había una diferencia de más de cinco puntos en el porcentaje de hombres y mujeres en situación de plena integración (51,4\% frente a 46,1\%), en 2013 los datos se nivelan hacia abajo para todos y todas (sólo el 34,2\% de los hombres y el 34,4\% de las mujeres están ya plenamente integrados). Lo mismo sucede en el caso de las personas en situación de integración precaria (donde la diferencia de casi tres puntos existente en 2007 desaparece en 2013) y de exclusión moderada (donde una diferencia de más de dos puntos en 2007 se reduce a 0,4 en 2013). Distinto es el caso de la exclusión severa, donde nunca ha habido diferencias significativas en función del sexo en el periodo analizado. Estos datos pueden sorprender, ya que muestran que las distancias entre hombres y mujeres, aunque más elevadas en 2007 que en 2013 , siempre han sido bastante reducidas. Este hecho, sin embargo, se explica por razones de orden metodológico: los datos de carácter individual aquí esgrimidos se han obtenido, de hecho, a partir de información agregada relativa al hogar -atribuyendo a todos sus miembros el mismo nivel de integración-, lo cual claramente limita la fluctuación.

El hecho de que las diferencias sean reducidas en las dos fechas consideradas no puede, por otra parte, llevarnos a ignorar que en 2013 lo son mucho más que en 2007, y que esta reducción se ha obtenido a costa de un empeoramiento generalizado de la situación social (en seis años, la plena integración disminuye un $33,5 \%$ en el caso de los hombres y un $25,4 \%$ en el caso de las mujeres). ¿Qué decir de esta evolución? ¿La lectura que de ella podemos hacer es, desde una perspectiva de género, positiva o negativa? Ambas opciones son posibles: por un lado, estos datos podrían reflejar una tendencia estable hacia la reducción de las desigualdades de género, lo cual sería claramente positivo; por otro, sin embargo, podría tratarse de algo puramente coyuntural, reflejo de una crisis que ha impactado más en sectores profesionales masculinizados. Esta última hipótesis es claramente negativa, sobre todo porque la constatación de una menor desigualdad entre géneros podría llegar a debilitar las políticas de igualdad, cuando en realidad su necesidad seguiría invariada.

Los datos hasta aquí presentados son significativos y útiles para obtener una primera fotografía de la realidad. En ellos, sin embargo, como ya se ha apuntado, las desigualdades de género resultan parcialmente invisibilizadas e infrarrepresentadas. Para comprender esta afirmación, hay que tener en cuenta que los datos de carácter individual presentados son, en realidad, datos de hogar, y que a todos los individuos que componen un hogar se atribuye automáticamente el mismo índice de exclusión social. Por un 


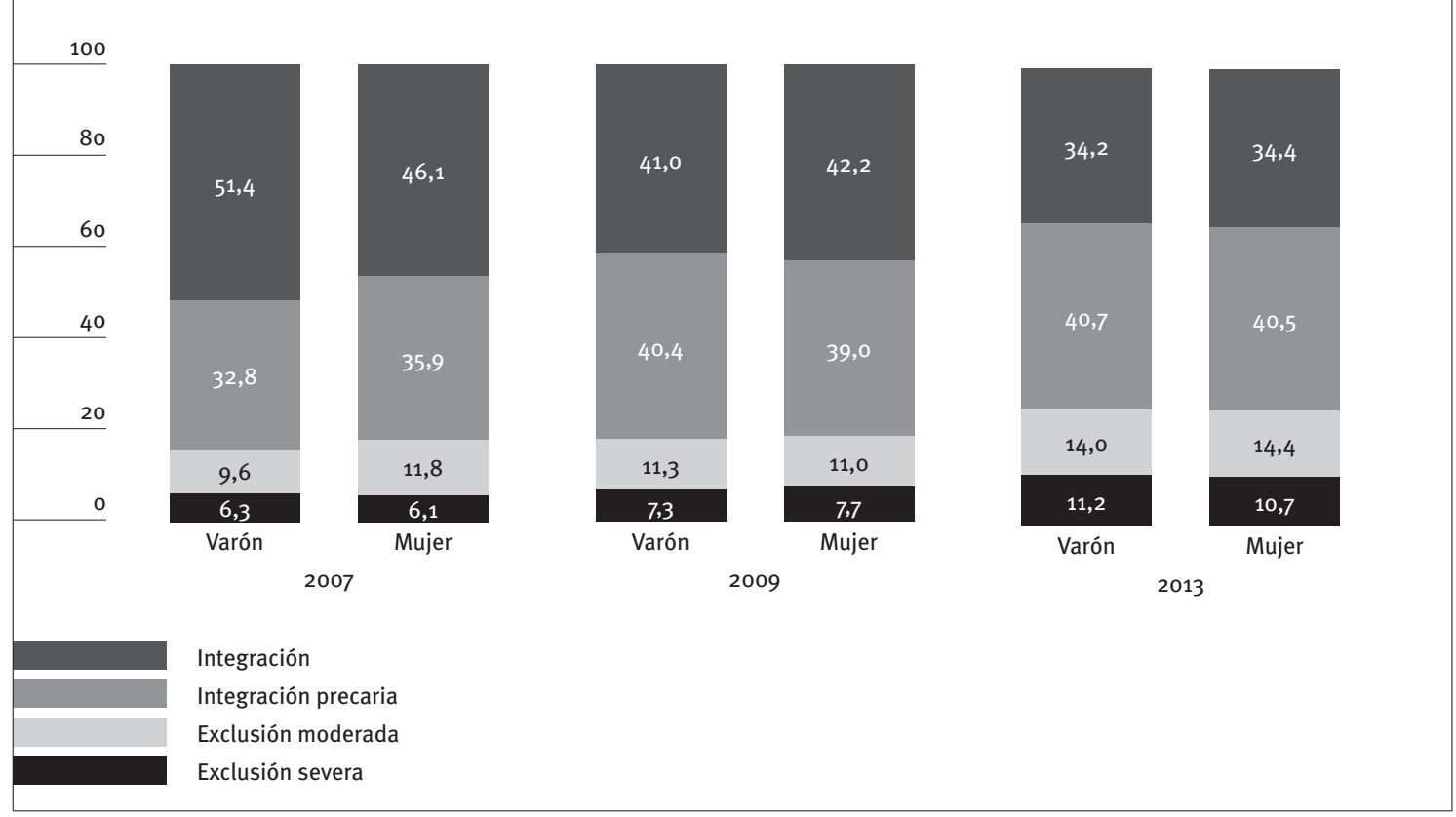

Fuente: Elaboración propia a partir de las Encuestas Foessa 2007, 2009 y 2013.

lado, esta decisión se justifica por el hecho de que los hogares constituyen unidades de consumo, de redistribución interna de recursos o de creación de solidaridades y apoyos (Laparra, 2014). Por otro, sin embargo, varios autores y autoras señalan que la distribución de los recursos en el seno de la familia está lejos de ser igualitaria y equitativa (Fernández Viguera, 1992 y 1998; Gaviria, Laparra y Aguilar, 1995; Gabàs, 2003).

Por ello, un acercamiento a las desigualdades existentes entre hombres y mujeres no puede limitarse al análisis que se ha realizado en el presente apartado, sino que requiere combinarlo con un análisis a nivel de hogares, comparando los casos en que el sustentador principal es un hombre con aquellos en que es una mujer.

\section{Exclusión social: permanecen las diferencias entre hogares}

El análisis por hogares muestra que, entre 2007 y 2013, el porcentaje de hogares encabezados por una mujer ha crecido 6,7 puntos (desde el 26,5\% en 2007 y 2009 hasta el 33,2 \% en 2013). Avanzamos la hipótesis de que este aumento está relacionado con una crisis económica que ha impactado con más fuerza en sectores masculinizados (in primis la construcción) [Laparra, 2013], lo cual ha convertido el sueldo de la mujer, antes secundario o incluso inexistente, en el sueldo principal del hogar. Esta sustitución, por otra parte, no consigue evitar un empeoramiento de las condiciones generales del hogar, y esto por varias razones: en primer lugar, porque sigue existiendo una brecha de género en el mercado laboral, que se concreta, en el caso de las mujeres, en salarios inferiores y en una mayor incidencia del empleo precario, discontinuo y a tiempo parcial (Fernández Viguera, 1998; Subirats, 2004; UGT Navarra, 2014); en segundo lugar, porque, en muchos casos, el sueldo de la mujer se concebía simplemente como complementario al del varón, con todo lo que esto implica (jornada parcial, empleos irregulares); finalmente, porque, en algunos hogares, el varón era antes el único sustentador, y si la mujer ingresaba en el mercado de trabajo era solamente porque aquél se quedaba en desempleo. Es fácil hipotetizar que, si una mujer que lleva años ausente del mercado laboral -a menudo, con cargas familiares- ingresa en él en época de crisis, difícilmente las condiciones de empleo serán buenas.

En relación con el nivel de integración/exclusión, dos son los hallazgos fundamentales del análisis por hogares: en primer lugar, observamos que, aunque la situación ha empeorado para todo el mundo, este empeoramiento es ligeramente más intenso en el caso de hogares cuyo sustentador principal es un hombre; en segundo lugar, detectamos que, pese a ello, en 2013 los hogares encabezados por mujeres siguen enfrentando un riesgo considerablemente más elevado de vivir procesos de exclusión.

Empezando por el primer punto, podemos observar que, entre 2007 y 2013 , el porcentaje de hogares en plena integración se ha reducido drásticamente, tanto para hogares encabezados por mujeres como por hombres, aunque algo más en el caso de estos últimos (9,9 puntos frente a 9,2). Paralelamente, se observa un incremento de los hogares en situación 


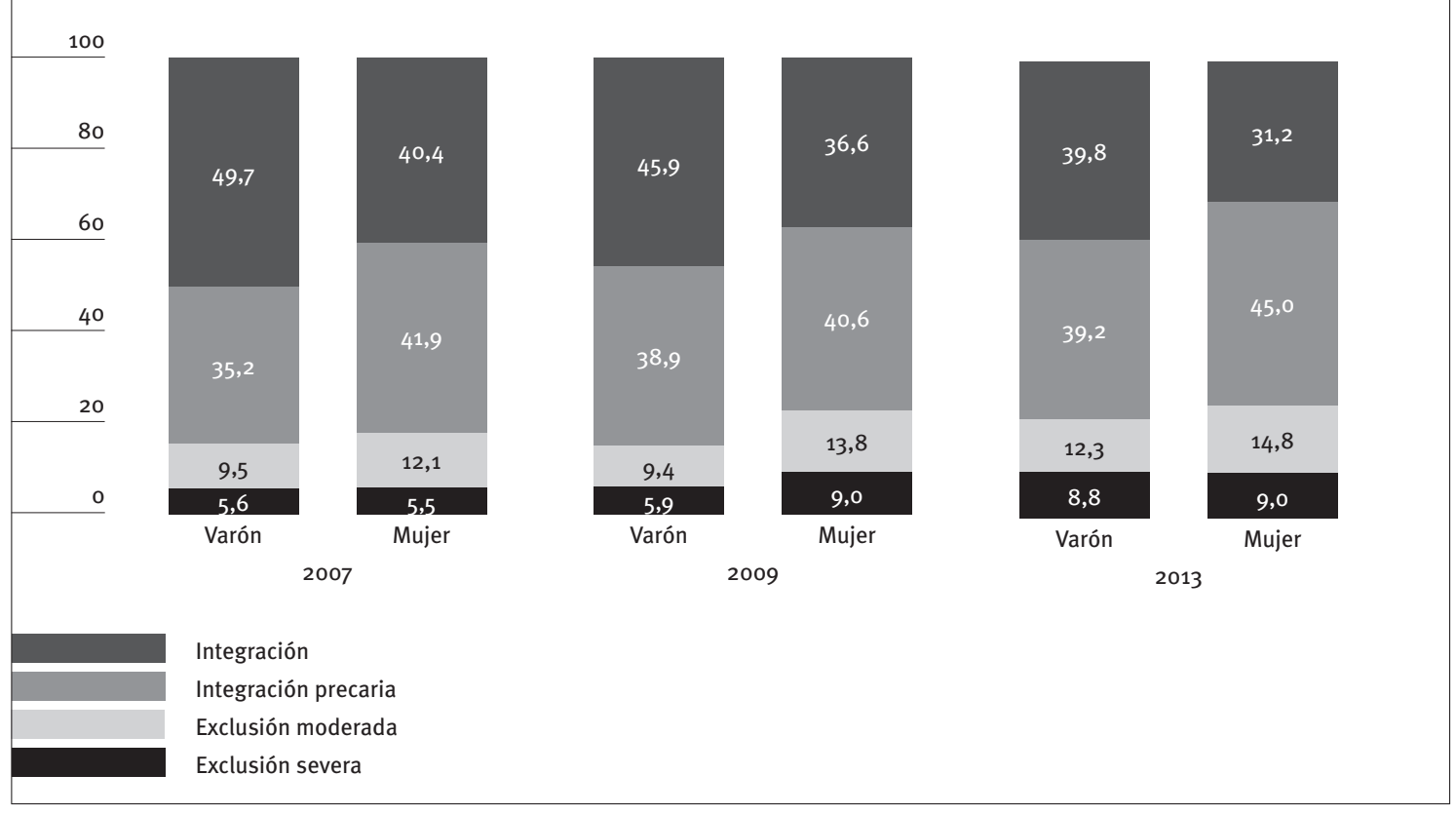

Fuente: Elaboración propia a partir de las Encuestas Foessa 2007, 2009 y 2013.

de integración precaria, exclusión moderada y exclusión severa. En el caso de la integración precaria, este aumento es generalizado, pero levemente más intenso para los hogares encabezados por hombres (4 puntos frente a 3,1) ${ }^{4}$. En lo referente a la exclusión moderada, no se aprecian diferencias significativas en la evolución en función del sexo (aumento de 2,8 y 2,7 puntos, respectivamente). Finalmente, en el caso de la exclusión severa, la situación se invierte y se observa un empeoramiento generalizado, pero más significativo entre los hogares encabezados por mujeres (que aumentan 3,5 puntos su presencia en la exclusión severa, frente a 3,2 puntos cuando el sustentador principal es un hombre). El ISES confirma estos resultados y muestra que, aunque el aumento es generalizado, lo es más entre los hogares encabezados por varones (de 1,15 a 1,39 cuando el sueldo principal es aportado por una mujer, y de 0,95 a 1,29 cuando es aportado por un varón). En otras palabras, las diferencias en el ISES entre unos y otras se reducen de 0,2 puntos en 2007 a 0,1 puntos en 2013.

En segundo lugar, observamos que, aunque la situación empeora más intensamente para los hogares encabezados por hombres, los encabezados por mujeres siguen enfrentando un riesgo de vivir procesos de exclusión considerablemente más

${ }^{4}$ Aunque la integración precaria se sitúa en el espacio de la integración, consideramos que, en un contexto de profunda crisis económica y de drástica reducción de los hogares en plena integración, el incremento del porcentaje de hogares en integración precaria debe ser interpretado en términos negativos, como el primer paso de una trayectoria descendente hacia la exclusión. Por el contrario, si el aumento de la integración precaria fuese asociado a una reducción de la exclusión, nuestra interpretación sería claramente distinta. elevado. En 2013, de hecho, el porcentaje de hogares en situación de plena integración era, en el caso de los hogares encabezados por hombres, 8,6 puntos más elevado que entre los encabezados por mujeres, es decir, había una diferencia del $27,6 \%$. Al contrario, el porcentaje de hogares en integración precaria era 5,8 puntos más elevado cuando la sustentadora principal era una mujer, lo cual se traduce en un riesgo 14,8 puntos porcentuales más elevado que el que enfrentan los hogares encabezados por varones. Lo mismo sucede en el caso de la exclusión moderada, donde las diferencias alcanzan los 2,5 puntos. En el caso de la exclusión severa, al contrario, no se evidencian diferencias significativas en función del sexo de la persona que más ingresos aporta al hogar, ni en 2007 ni en 2013. Nuevamente, el ISES confirma nuestros análisis y muestra que sigue existiendo una situación de desventaja comparativa en los hogares encabezados por mujeres (ISES igual a 1,39, frente a 1,29 cuando el sueldo principal es aportado por un varón).

Resumiendo, los datos muestran con claridad que los hogares encabezados por mujeres presentan un mayor riesgo de vivir procesos de exclusión. Por otra parte, si limitamos el análisis a los hogares en situación de exclusión (tanto moderada como severa) descubrimos que, en las tres fechas analizadas, los hogares excluidos encabezados por un varón experimentan una exclusión más intensa (ISES más elevado) que los hogares excluidos encabezados por una mujer. Esta distancia ha ido progresivamente aumentando en los últimos años, pasando de 0,2 puntos (4,2 frente a 4,4) en 2007 a 0,3 en $2009(4,1$ frente a 4,4) y 0,4 en $2013(4,6$ frente a 4,2). 


\section{Dimensiones e indicadores de los procesos de exclusión}

Una vez realizado este análisis por hogares, acercamos la mirada y mostramos cómo las diferencias entre hombres y mujeres no atañen sólo a los aspectos cuantitativos -incidencia del fenómeno-, sino también a factores más 'cualitativos'-importancia relativa de las distintas dimensiones de aquél-. Para ello, realizaremos un análisis en tres niveles, observando las diferencias entre hogares en relación a:

- Los tres ejes básicos de los procesos de exclusión (económico, político y social-relacional) [Gráfico 3].

- Las siete dimensiones en las que éstos se concretan (exclusión del empleo, del consumo, de la participación política, de la educación, de la vivienda, de la salud, conflicto social y aislamiento social) [Tabla 1].

- Los 35 indicadores que permiten identificar la existencia de exclusión en dichas dimensiones [Tabla 2].

En primer lugar, consideramos los tres ejes que conforman las situaciones de exclusión. El primero, el eje económico, identifica las situaciones de exclusión del empleo (tanto por la falta de éste como por sus características) y del consumo. El segundo, el eje político, mide la falta de participación política y de acceso a los derechos de ciudadanía social (educación, vivienda digna y cobertura sanitaria). El tercero, el eje socialrelacional, identifica, por un lado, las situaciones de aislamiento social y, por otro, la integración en redes sociales 'perversas' (Laparra y Pérez, 2010). Tal y como se puede apreciar en el Gráfico 2, aunque en las tres dimensiones el riesgo es mayor para los hogares encabezados por una mujer, en las tres fechas analizadas la intensidad de las diferencias entre mujeres y hombres varía profundamente según el eje considerado.

En primer lugar, podemos observar cómo, tanto en 2007 como en 2009 y 2013 , las mayores diferencias atañen a la esfera social-relacional. En 2013, por ejemplo, el riesgo de enfrentar exclusión relacional era, para los hogares encabezados por una mujer, más del doble que entre los hogares donde el sueldo principal era aportado por un varón (17,5\% frente a $7,8 \%$ ) [Gráfico 3]. Esos mayores niveles de exclusión relacional indicaban una mayor incidencia tanto de conflicto social $(8,3 \%$ frente a $4,2 \%)$ como de aislamiento $(9,7 \%$ frente a $4,1 \%)$ [Tabla 1]. En relación con

Tabla 1. Distribución de los hogares que experimentan exclusión, en sus distintas dimensiones, según el sexo de la persona sustentadora principal. España, 2007, 2009 y 2013 (\%)

\begin{tabular}{|c|c|c|c|c|c|c|}
\hline & \multicolumn{2}{|c|}{2007} & \multicolumn{2}{|c|}{2009} & \multicolumn{2}{|c|}{2013} \\
\hline & Varón & Mujer & Varón & Mujer & Varón & Mujer \\
\hline Exclusión del empleo & 15,4 & 17,1 & 23,5 & 24,2 & 33,5 & 35,8 \\
\hline Exclusión del consumo & - & - & - & - & 5,4 & 6,9 \\
\hline Exclusión política & 12,7 & 14,9 & 19,0 & 21,1 & 12,4 & 13,4 \\
\hline Exclusión de la educación & 11,2 & 9,0 & 10,6 & 12,7 & 7,6 & 9,4 \\
\hline Exclusión de la vivienda & 18,5 & 25,3 & 19,1 & 20,9 & 24,1 & 28,3 \\
\hline Exclusión de la salud & 10,1 & 15,9 & 8,8 & 14,9 & 17,0 & 20,9 \\
\hline Conflicto social & 5,2 & 4,6 & 5,3 & 7,9 & 4,2 & 8,3 \\
\hline Aislamiento social & 6,2 & 12,1 & 3,7 & 12,4 & 4,1 & 9,7 \\
\hline
\end{tabular}

Fuente: Elaboración propia a partir de las Encuestas Foessa 2007, 2009 y 2013.

Gráfico 3. Distribución de los hogares que experimentan exclusión, en sus distintas dimensiones, según el sexo de la persona sustentadora principal. España, 2007, 2009 y 2013 (\%)

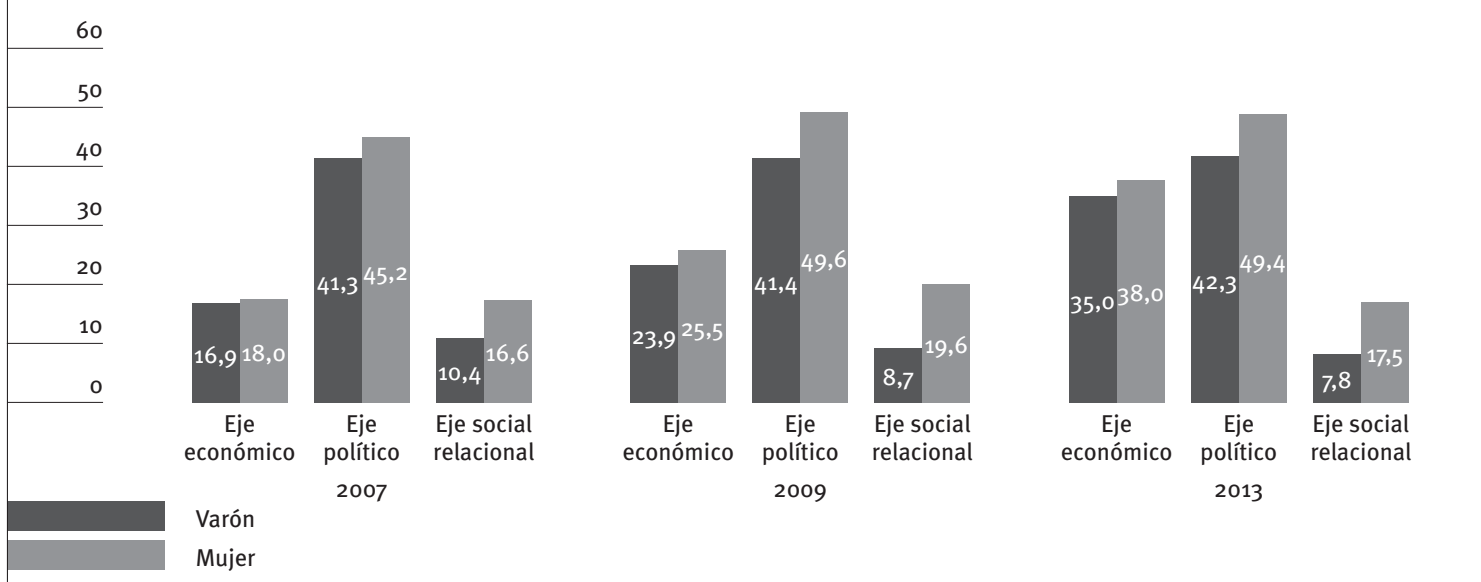

Fuente: Elaboración propia a partir de las Encuestas Foessa 2007, 2009 y 2013. 
Tabla 2. Hogares que presentan cada uno de los indicadores de exclusión, según el sexo de la persona sustentadora principal. España, 2007, 2009 y 2013 (\%)

\begin{tabular}{|c|c|c|c|c|c|c|}
\hline \multirow{3}{*}{ Dimensiones } & \multirow{3}{*}{ Aspectos } & \multirow{3}{*}{ Indicadores } & \multicolumn{4}{|c|}{ Incidencia } \\
\hline & & & \multicolumn{2}{|c|}{2007} & \multicolumn{2}{|c|}{2013} \\
\hline & & & Varón & Mujer & Varón & Mujer \\
\hline \multirow{6}{*}{$\begin{array}{l}\text { Participación } \\
\text { de la } \\
\text { producción }\end{array}$} & \multirow{6}{*}{ Empleo } & Sustentador/a principal en paro desde hace al menos un año & 3,3 & 4,7 & 10,9 & 10,1 \\
\hline & & Sustentador/a principal con un oficio de exclusión & 2,9 & 6,9 & 1,7 & 5,4 \\
\hline & & $\begin{array}{l}\text { Sustentador/a principal con empleo irregular, sin cobertura de la } \\
\text { Seguridad Social }\end{array}$ & 5,6 & 5,7 & 2,3 & 3,4 \\
\hline & & $\begin{array}{l}\text { Sin miembros ocupados, ni pensionistas contributivos, ni de baja, ni } \\
\text { con prestaciones contributivas por desempleo }\end{array}$ & 10,4 & 12,8 & 10,9 & 12,7 \\
\hline & & Algún miembro parado y sin formación ocupacional & 17,6 & 14,5 & 31,3 & 30,8 \\
\hline & & Todos los miembros activos en paro & 8,5 & 9,8 & 19,9 & 19,9 \\
\hline \multirow{2}{*}{$\begin{array}{l}\text { Participación } \\
\text { del producto } \\
\text { social }\end{array}$} & Ingresos & $\begin{array}{l}\text { Pobreza extrema (ingresos inferiores al } 30 \% \text { de la renta familiar } \\
\text { mediana equivalente) }\end{array}$ & 7,4 & 7,9 & 6,5 & 8,0 \\
\hline & Privación & $\begin{array}{l}\text { Privación de al menos un bien básico (agua corriente, agua caliente, } \\
\text { energía eléctrica, evacuación de aguas sucias, baño completo, cocina, } \\
\text { frigorífico, lavadora) }\end{array}$ & - & - & 1,9 & 2,6 \\
\hline \multirow{2}{*}{$\begin{array}{l}\text { Derechos } \\
\text { políticos }\end{array}$} & \multirow{2}{*}{$\begin{array}{l}\text { Participación } \\
\text { política }\end{array}$} & $\begin{array}{l}\text { Sin derecho a elegir a representantes políticos y a ser elegido/a como } \\
\text { tal (hogares con alguna persona mayor de } 17 \text { años de nacionalidad } \\
\text { extracomunitaria) }\end{array}$ & 9,4 & 8,6 & 6,4 & 4,9 \\
\hline & & $\begin{array}{l}\text { Sin capacidad efectiva de ser considerado/a y de influir en el proceso } \\
\text { de toma de decisiones colectivas (no participan en las elecciones por } \\
\text { falta de interés y no son miembros de ninguna entidad ciudadana) }\end{array}$ & 6,7 & 8,2 & 8,2 & 11,1 \\
\hline \multirow{25}{*}{$\begin{array}{l}\text { Derechos } \\
\text { sociales }\end{array}$} & \multirow{3}{*}{ Educación } & Miembros de 3 a 15 años no escolarizados & 1,9 & 1,0 & 1,4 & 1,0 \\
\hline & & $\begin{array}{l}\text { Ningún miembro de } 16 \text { a } 64 \text { años con estudios (de } 16 \text { a } 44 \text {, sin } \\
\text { completar EGB, ESO o graduado escolar; de } 45 \text { a } 64 \text { años, menos de } 5 \\
\text { años en la escuela) }\end{array}$ & 9,6 & 7,8 & 5,3 & 4,8 \\
\hline & & $\begin{array}{l}\text { Algún miembro de más de } 64 \text { años que no sabe leer y escribir (o no ha } \\
\text { ido a la escuela) }\end{array}$ & 8,5 & 10,8 & 3,9 & 6,2 \\
\hline & \multirow{8}{*}{ Vivienda } & Infravivienda (chabola, bajera, barracón, prefabricado o similar) & 1,8 & 1,5 & 1,1 & 0,7 \\
\hline & & Deficiencias graves en la construcción, ruina, etc. & 4,3 & 5,0 & 2,0 & 1,5 \\
\hline & & Humedades, suciedad y olores (insalubridad) & 18,2 & 16,8 & 8,6 & 10,2 \\
\hline & & Hacinamiento grave ( $\left(15 \mathrm{~m}^{2} /\right.$ persona $)$ & 8,4 & 4,5 & 4,9 & 4,3 \\
\hline & & $\begin{array}{l}\text { Tenencia en precario (facilitada gratuitamente por otras personas o } \\
\text { instituciones, realquilada, ocupada ilegalmente) }\end{array}$ & 5,1 & 4,2 & 1,2 & 1,7 \\
\hline & & Entorno muy degradado & 2,8 & 1,8 & 2,3 & 2,0 \\
\hline & & $\begin{array}{l}\text { Barreras arquitectónicas con personas con discapacidad física en el } \\
\text { hogar }\end{array}$ & 4,3 & 6,5 & 5,7 & 5,0 \\
\hline & & $\begin{array}{l}\text { Gastos excesivos de la vivienda (ingresos - gastos vivienda < umbral } \\
\text { pobreza extrema) }\end{array}$ & 11,0 & 12,1 & 11,3 & 16,2 \\
\hline & \multirow{6}{*}{ Salud } & Algún miembro sin cobertura sanitaria & 0,9 & 0,4 & 0,3 & 0,1 \\
\hline & & Pasan hambre con frecuencia o lo han hecho en los últimos diez años & 2,9 & 4,4 & 2,2 & 5,2 \\
\hline & & $\begin{array}{l}\text { Todos los miembros adultos con minusvalía, enfermedad crónica } \\
\text { o problemas graves de salud que les generan limitaciones para las } \\
\text { actividades de la vida diaria }\end{array}$ & 1,9 & 5,6 & 1,9 & 5,0 \\
\hline & & $\begin{array}{l}\text { Miembros dependientes (que necesitan ayuda o cuidados de otras } \\
\text { personas para realizar las actividades de la vida diaria y que no la } \\
\text { tienen) }\end{array}$ & 1,4 & 3,3 & 1,4 & 1,5 \\
\hline & & $\begin{array}{l}\text { Miembros enfermos/as que no han usado los servicios sanitarios en } \\
\text { un año }\end{array}$ & 1,3 & 2,2 & 1,0 & 0,9 \\
\hline & & $\begin{array}{l}\text { Han dejado de comprar medicinas, seguir tratamientos o dietas por } \\
\text { problemas económicos }\end{array}$ & 13,0 & 16,8 & 12,2 & 15,3 \\
\hline & \multirow{2}{*}{$\begin{array}{l}\text { Conflictos } \\
\text { familiares }\end{array}$} & $\begin{array}{l}\text { Algún miembro que recibe malos tratos físicos o psicológicos, o los ha } \\
\text { recibido en los últimos diez años }\end{array}$ & - & - & 1,5 & 5,5 \\
\hline & & Relaciones muy malas, malas o más bien malas & 1,5 & 1,6 & 0,6 & 0,8 \\
\hline & \multirow{2}{*}{$\begin{array}{l}\text { Conductas } \\
\text { asociales }\end{array}$} & $\begin{array}{l}\text { Miembros que tienen problemas con el alcohol, otras drogas o con el } \\
\text { juego, o los han tenido en los últimos diez años }\end{array}$ & - & - & 2,8 & 3,0 \\
\hline & & $\begin{array}{l}\text { Algún miembro a punto de ser madre adolescente sin pareja, o que ya } \\
\text { lo ha sido }\end{array}$ & 3,1 & 3,0 & 0,6 & 1,4 \\
\hline & $\begin{array}{l}\text { Conductas } \\
\text { delictivas }\end{array}$ & $\begin{array}{l}\text { Miembros que tiene problemas con la justicia (antecedentes penales), } \\
\text { o los han tenido en los últimos diez años }\end{array}$ & 3,7 & 5,2 & 1,2 & 1,6 \\
\hline & Sin apoyo familiar & $\begin{array}{l}\text { Miembros que carecen de relaciones y que no cuentan con ningún } \\
\text { apoyo para situaciones de enfermedad o de dificultad }\end{array}$ & 3,6 & 12,1 & 3,2 & 10,1 \\
\hline & Conflicto vecinal & Malas o muy malas relaciones con los vecinos & 1,2 & 0,3 & 0,4 & 0,6 \\
\hline & \begin{tabular}{l|} 
Personas \\
institucionalizadas
\end{tabular} & $\begin{array}{l}\text { Algún miembro en instituciones (hospitales y pisos psiquiátricos, } \\
\text { centros de drogodependencias, de menores, penitenciarios, para } \\
\text { transeúntes o mujeres) }\end{array}$ & 0,7 & 1,4 & 0,3 & 0,2 \\
\hline
\end{tabular}

Fuente: Elaboración propia a partir de las Encuestas Foessa 2007 y 2013. 
el primero, cabe destacar que, aunque los indicadores a partir de los cuales se deduce la presencia de conflicto son varios, sólo uno puede explicar la existencia de una distancia tan grande entre hogares encabezados por hombres y por mujeres: nos referimos al porcentaje de hogares donde algún miembro ha experimentado o experimenta malos tratos $(5,5 \%$ cuando la sustentadora principal es una mujer y $1,5 \%$ cuando es un hombre) [Tabla 2]. Estas diferencias en función del género sugieren que una gran parte de este maltrato identifica situaciones de violencia de género. Más específicamente, se trataría de mujeres expulsadas de sus hogares por maltrato y que, a consecuencia de eso, son en la actualidad las sustentadoras principales de su propio hogar.

En relación con el aislamiento social, por otra parte, las diferencias en función del sexo de la persona que aporta más ingresos al hogar son el resultado de una incidencia considerablemente mayor, entre hogares encabezados por una mujer, de la falta de apoyo familiar. Así, mientras que sólo 3,2 \% de los hogares encabezados por un varón experimentan ausencia de relaciones en el hogar y falta de apoyo para momentos de dificultad, este porcentaje asciende al 10,1\% (es decir, más del triple) cuando la sustentadora principal es una mujer (Tabla 2). Si consideramos que este tipo de situaciones afectan mayoritariamente a personas mayores que viven solas, entonces la mayor incidencia entre hogares encabezados por una mujer se podría (en parte) explicar a partir de la mayor esperanza de vida femenina, que deja un porcentaje de viudas mucho más elevado que de viudos. Tal hipótesis se confirmaría si consideramos que, entre los hogares que carecen de apoyo familiar, cuando el sustentador principal es un hombre solamente, el $33,3 \%$ tiene más de 65 años, mientras que el porcentaje asciende hasta el $61,1 \%$ cuando es una mujer.

En segundo lugar, observamos la esfera política. En este caso, las diferencias entre hogares en función del sexo de la persona que aporta más ingresos al hogar se sitúan en un lugar intermedio: alcanzan los siete puntos ( $49,4 \%$ cuando la sustentadora principal es una mujer y $42,3 \%$ cuando es un hombre), una distancia mayor que en el caso de la esfera económica (donde no superan los tres), pero inferior que en la esfera relacional, (donde, como hemos visto, llegaban casi a diez puntos) [Gráfico 3]. Aquí, el panorama se muestra más confuso que en la esfera relacional: por un lado, los hogares encabezados por una mujer se muestran en desventaja en las tres dimensiones de educación, vivienda y sanidad; por otro, sin embargo, en todas estas dimensiones también aparecen indicadores donde los hogares encabezados por una mujer están mejor situados que los encabezados por un varón.

Las mayores diferencias se aprecian en el acceso a la vivienda, donde la distancia alcanza los 4,2 puntos $(28,3 \%$ cuando la sustentadora principal es una mujer, frente a $\mathbf{2 4 , 1} \%$ cuando es un varón) [Tabla 1]. El peor posicionamiento de los hogares encabezados por una mujer se deriva aquí, sobre todo, de la exis- tencia de gastos excesivos para la vivienda (16,2\% frente a 11,3\%) [Tabla 2]. Es lógico suponer que este gasto excesivo es, en realidad, el resultado de un sueldo insuficiente, hipótesis que se vería respaldada por la existencia de una brecha salarial entre mujeres y hombres. En otros casos, al contrario, las diferencias son más reducidas o llegan incluso a invertirse, como sucede en lo relativo a la infravivienda [Tabla 2]. Distancias elevadas se registran también en relación a la exclusión de la salud, donde la diferencia entre hogares encabezados por mujeres y por hombres alcanza los 3,9 puntos (20,9\% frente a $17 \%$ ) [Tabla 1]. En este caso, los indicadores clave son dos: uno identifica situaciones donde todos los miembros del hogar son dependientes ( $5 \%$ frente a $1,9 \%$ ) y otro mide las situaciones en que se ha pasado hambre $(5,2 \%$ frente a $2,2 \%)$ [Tabla 2]. Con respecto a la primera de las diferencias, al igual que en el caso del aislamiento, se explican considerando que, como efecto de la mayor longevidad femenina, el número de mujeres mayores que viven solas es superior al de los varones. Con respecto a la segunda, la explicación reside seguramente en los mayores niveles de pobreza femenina.

Una tercera dimensión es la exclusión educativa: aquí las diferencias son más reducidas en términos absolutos (9,4\% frente a 7,6\%), pero más elevadas en términos relativos (alcanzan casi el $20 \%$ ) [Tabla 1]. De nuevo, las diferencias clave apuntan a un efecto de la variable edad: las mayores distancias, de hecho, se registran entre hogares donde hay alguna persona mayor que no está alfabetizada (6,2 \% frente a 3,9\%) [Tabla 2]. En este caso, cabe destacar dos elementos: por un lado, vuelve a desplegar sus efectos la mayor longevidad femenina; por otro, no podemos ignorar que, mientras que en la actualidad las mujeres han alcanzado y superado a sus compañeros en términos de nivel educativo, esto no era así hace unas décadas. En suma, se dejarían todavía sentir los efectos de una menor escolarización de las mujeres en la primera mitad del siglo pasado.

En tercer lugar, terminamos esta breve presentación de las distintas dimensiones de los procesos de exclusión observando qué sucede con la esfera económica (Gráfico 3). En este caso, diferenciamos entre exclusión del empleo (que afecta al $38 \%$ de los hogares cuyo sueldo principal es aportado por una mujer y al $35 \%$ de los que tienen un sustentador principal varón) y del consumo (que afecta al 6,9\% y $5,4 \%$, respectivamente) [Tabla 1 ].

Con respecto a la primera, cabe destacar que, mientras que en 2007 se registraba una mayor incidencia del desempleo cuando la sustentadora principal era mujer (4,7\% frente a 3,3\%), en 2013 estas diferencias desaparecen, e incluso se invierten (10,9\% cuando el sueldo principal es aportado por un hombre y $10,1 \%$ cuando es aportado por una mujer) [Tabla 2]. Este cambio, como otros que ya se han analizado, está relacionado con un mayor impacto de la crisis en sectores masculinizados. Cabe también resaltar que se trata de una igualación a la baja, que, como ya se 
ha explicado con anterioridad, no favorece a nadie. Por otra parte, es interesante remarcar que, incluso en este contexto de igualación a la baja, si no observamos la cantidad de empleo, sino sus condiciones, descubrimos que la desventaja femenina permanece. Las mujeres, en efecto, trabajan en oficios de exclusión en un porcentaje mucho mayor que los hombres: en empleos que, por sus características, cabe considerar de exclusión, los valores son tres veces más elevados en el caso de las mujeres (5,4\% frente a 1,7\%); y en el caso de empleos sin cobertura de la Seguridad Social, las distancias, aunque más reducidas, sigues siendo significativas (3,4\% frente a 2,3\%) [Tabla 2]. Esta realidad se explica a partir de dos procesos distintos, pero relacionados. Por un lado, ya antes de la crisis existía una gran desigualdad en las condiciones de acceso al empleo (el 6,9\% de las sustentadoras principales trabajaban en un oficio de exclusión, frente al 2,9\% de los hombres) y ésta simplemente la ha perpetuado (Tabla 1). Por otro, en este periodo un gran número de mujeres ha querido entrar en el mercado laboral (la tasa de actividad femenina se ha incrementado casi cinco puntos en los últimos siete años, según la Encuesta de la Población Activa) para intentar suplir el desempleo de sus parejas masculinas. Su ingreso, sin embargo, como se ha apuntado más arriba, ha tenido lugar en una época de crisis, recorte y empeoramiento de las condiciones del empleo y ha venido, por lo tanto, marcado por estas circunstancias. En suma, la situación actual es el resultado de la interacción entre factores de tipo estructural y coyuntural.

En cuanto a la exclusión del consumo, solamente queremos destacar que el riesgo de vivir tanto la pobreza extrema como la privación de algún bien básico es mayor para los hogares encabezados por una mujer, y que las diferencias en función del sexo parecen estar creciendo, aunque ligeramente (de 1,5 a 1,7 puntos en el primer caso y de 0,5 a 0,7 en el segundo) [Tabla 2].

En conclusión, podemos afirmar que, aunque los hogares cuya sustentadora principal es una mujer enfrentan un riesgo de exclusión más elevado en cada una de las tres esferas consideradas, un examen más pormenorizado permite afinar el análisis. Así, nos muestra que las mayores diferencias atañen a la esfera social-relacional, seguida por la esfera política y, solamente en último lugar, por la económica. También cabe destacar que un análisis por dimensiones no es suficiente para dibujar una imagen precisa de las desigualdades, sino que es necesario integrarlo con un estudio de los indicadores que conforman cada dimensión. Así, por ejemplo, los mayores niveles de conflicto social responden sobre todo a la presencia de malos tratos, mientras que el alto riesgo de aislamiento esconde la influencia de la variable edad; en el caso de la esfera política, las diferencias encontradas se derivan sobre todo de las desigualdades en salud, educación y vivienda, mientras que no se hallan diferencias significativas en el nivel de participación política; finalmente, las diferencias en la esfera económica son un efecto sobre todo de la menor calidad del empleo femenino y los mayores niveles de pobreza de las mujeres. Por medio de un análisis de este tipo, podemos establecer dónde se encuentran las claves de la desigualdad, comprender mejor los procesos de exclusión, y mejorar así la planificación de proyectos de intervención y políticas públicas.

\section{La esfera laboral y las desigualdades internas al hogar}

Una vez ofrecida una panorámica general de las distintas dimensiones de los procesos de exclusión, centramos la mirada en la esfera económica, y en concreto, en cómo las desigualdades de género en el acceso al mercado laboral tienen consecuencias de cara a los procesos de exclusión. La determinación de dedicar un apartado específico a este análisis se explica por dos razones. En primer lugar, porque aun siendo la exclusión un fenómeno multidimensional, que va más allá de la esfera económica y laboral (Laparra et al., 2007), en la sociedad actual el empleo constituye uno de los factores de integración más importantes. En segundo lugar, porque el laboral es un sector donde las diferencias entre hombres y mujeres perduran en toda su intensidad.

Descubrimos así que tanto el número de personas empleadas como su sexo tienen un claro efecto en el riesgo de vivir procesos de exclusión, por un lado, y en su intensidad, por otro. Más en detalle, podemos ver que los mayores niveles de exclusión (55,6\%) se dan en ese $16 \%$ de hogares donde no trabaja ninguno de los cónyuges. Es también ahí donde, dentro del espacio de exclusión, es mayor el peso de la exclusión severa, en la que se encuentran el $57,4 \%$ de los hogares excluidos. Por el contrario, los niveles menores de exclusión (11,5\%) se registran en ese $39,3 \%$ de hogares donde ambos miembros tienen un empleo. Es más, no solamente el riesgo de exclusión es infinitamente menor, sino que aquí, a diferencia que en el caso anterior, dentro del espacio de exclusión prima la exclusión moderada, en la que se encuentra más del $60 \%$ de los hogares excluidos.

Para un análisis de género, sin embargo, resulta más interesante observar los hogares donde trabaja solamente uno de los miembros de la pareja y, en particular, las diferencias que se dan en el nivel de integración según que la persona empleada sea una mujer o un hombre. Podemos así observar que el género tiene consecuencias muy profundas en el riesgo de exclusión: en el $14 \%$ de hogares en los que la persona empleada es una mujer, el riesgo de exclusión más que duplica el de ese $30 \%$ de hogares donde sólo tiene empleo el varón ( $41,3 \%$ frente a 20,9\%)

Para comprender e interpretar estos datos, debemos remitirnos a diversos elementos. Por un lado, debemos tener en cuenta la existencia de una importante brecha salarial entre mujeres y hombres, que en 2012 alcanzaba el 17,8\% (según Eurostat) y que segura- 


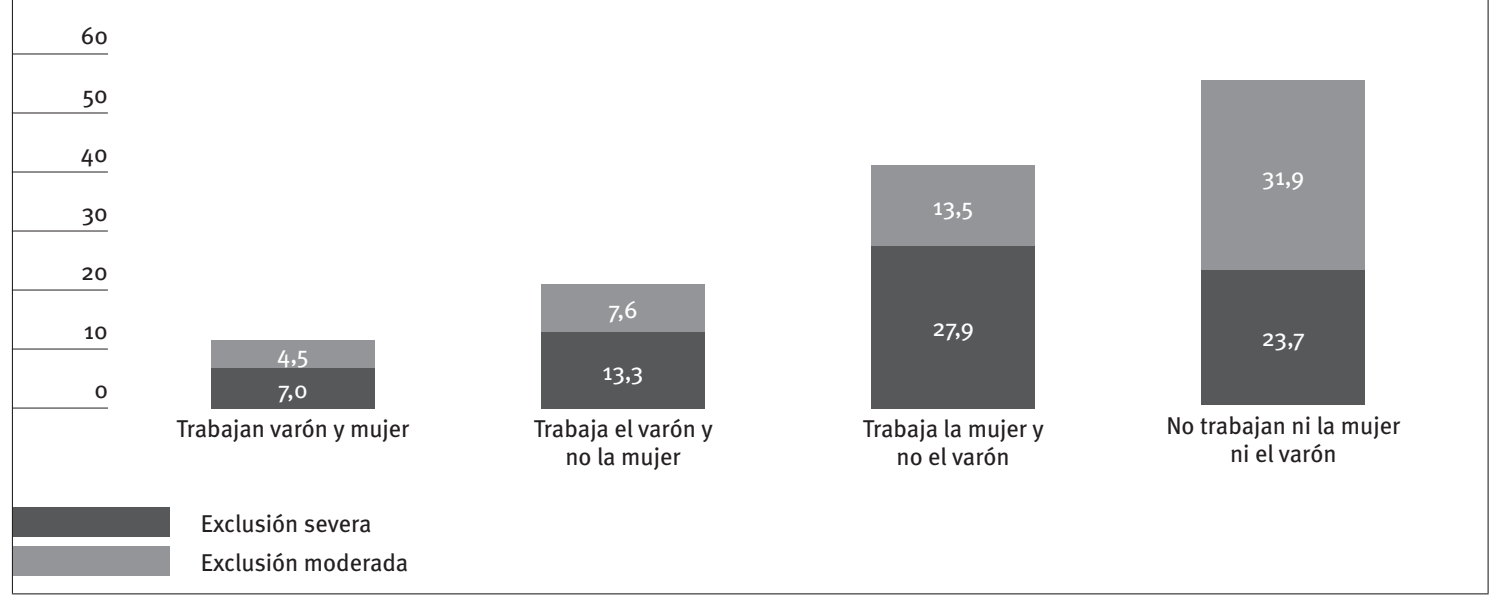

Fuente: Elaboración propia a partir de la Encuesta Foessa 2013.

mente favorece la existencia de situaciones de exclusión económica cuando la única persona empleada es la mujer. Por otro lado, también es necesario tener en cuenta que las motivaciones que llevan a estos hogares a ser sustentados únicamente por una persona suelen ser distintas según ésta sea un varón o una mujer. El primer caso, de hecho, responde a una estructura clásica de división sexual del trabajo -el modelo del varón proveedor (Izquierdo, 2010)-. Es, por lo tanto, lógico hipotetizar que el hecho de que solamente el varón tenga empleo es el resultado de una decisión 'libremente's tomada por los dos miembros de la pareja e independiente de los efectos de la crisis económica. La segunda situación, al contrario, nos lleva a suponer que la gran mayoría de los casos responden a situaciones no elegidas, sino provocadas por la pérdida de empleo por parte del varón. Esto podría haber dado lugar tanto a situaciones en la que un sueldo antes secundario se ha convertido en único ingreso como a otras donde la mujer ha ingresado en el mercado de trabajo precisamente para suplir la expulsión del varón de aquél. Como ya se ha indicado, ambas situaciones incrementan el riesgo de que el empleo sea de baja calidad.

\section{Colectivos en situación de mayor vulnerabilidad}

Una vez analizada la situación de la generalidad de los hogares, centramos la mirada en aquellos que pertenecen a colectivos de riesgo (es decir, donde la probabilidad de que se den procesos de exclusión es mayor). Nos referimos, en particular, a los hogares con algún miembro de origen extracomunitario o de la ampliación de la UE, a los hogares cuyo/a

${ }^{5}$ Las comillas responden al hecho de que, aunque la decisión no es efecto de la crisis económica, tampoco es totalmente libre, ya que responde a unos condicionantes estructurales que asignan al varón las tareas productivas, y a la mujer, las tareas reproductivas y de cuidados. sustentador/a principal tiene menos de 30 años, y a los hogares monoparentales. En todos los casos, como veremos, se registra una clara tendencia a la igualación entre hombres y mujeres.

\subsection{Hogares con algún miembro de origen extraeuropeo}

En relación a los hogares con algún miembro de origen extracomunitario o de la ampliación de la UÉ, empezamos destacando que, entre 2007 y 2013 , su importancia numérica ha disminuido (desde el $11,4 \%$ en 2007 al $10,8 \%$ en 2009 y el $9,2 \%$ en 2013 ), seguramente como efecto del retorno de una parte importante de la población inmigrante a sus países de origen, por un lado, y de los procesos de nacionalización, por otro. En segundo lugar, observamos que en esos hogares, al igual que para el conjunto de los hogares españoles, ha aumentado el porcentaje de casos donde el sueldo principal es aportado por una mujer, pasando del $23,6 \%$ en 2007 al 32,6\% en 2013, es decir, un incremento de 9 puntos. Como ya aclaramos en el caso de la generalidad de los hogares, aquí también avanzamos la hipótesis de que este aumento está relacionado con un mayor impacto de la crisis en sectores masculinizados (in primis la construcción) [Laparra, 2013].

En cuanto a la evolución de la exclusión entre 2007 y 2013, se evidencia una clara diferencia entre el conjunto de los hogares y aquellos con algún miembro de origen extracomunitario o de la ampliación de la UE. En el primer caso, de hecho, los hogares encabezados por hombres ven simplemente reducir su ventaja, manteniendo sin embargo una clara distancia con respecto a los hogares encabezados por mujeres. En el segundo caso, al contrario, la situación no ha

${ }^{6}$ Se consideran aquí las personas originarias de países no miembros de la Unión Europea o de países de reciente incorporación a ésta (es decir, todos aquellos que no formaban parte de la UE12). 
cambiado sólo cuantitativamente, sino también cualitativamente: mientras que en 2007 la diferencia en función del sexo era extremadamente intensa (los hogares encabezados por mujeres tenían un riesgo más que doble de vivir procesos de exclusión), en 2013 se había invertido y era 4,4 puntos menores. El ISES también da cuenta de esta inversión, y pasa de ser un $78 \%$ más elevado cuando la sustentadora principal es una mujer en 2007 a ser un $16 \%$ más reducido en 2013 (2,91 frente a 2,44).

Por otra parte, no podemos olvidar que esta reducción de las desigualdades de género se ha obtenido a costa de un empeoramiento general e intenso de la situación de los hogares con algún miembro de origen extranjero, que entre 2007 y 2013 han visto su ISES aumentar un $56 \%$ (del 1,77 en 2007 al 2,76 en 2013).

Gráfico 5. Distribución de los hogares con algún miembro de origen extracomunitario o de la ampliación de la UE en situación de exclusión, según el sexo de la persona sustentadora principal. España, 2007 y 2013 (\%)
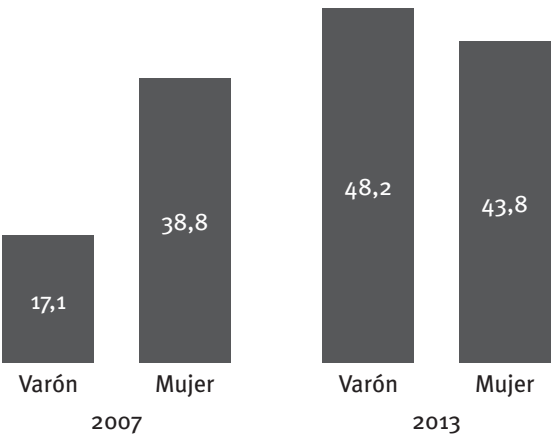

Fuente: Elaboración propia a partir de las Encuestas Foessa 2007 y 2013.

Si afinamos el análisis y observamos cómo se distribuyen los hogares en los cuatro espacios de integración/ exclusión, por otra parte, observamos que los encabezados por una mujer, aunque tienen muchas probabilidades de situarse en la zona de integración, se sitúan mayoritariamente en la de integración precaria $(49,1 \%$ frente a $42 \%$ ), mientras que la integración plena sigue siendo un espacio principalmente masculino (9,9\% frente a 7,1\%). Al contrario, si observamos el espacio de la exclusión, observamos que los hogares cuyo sueldo principal es aportado por un varón, no solamente tienen mayores probabilidades de encontrarse en aquélla, sino que en su interior tienden mucho más a concentrarse en la exclusión severa (cuando el sustentador principal es un varón, el 53,6\% de los hogares excluidos se encuentran en exclusión severa, valor que se reduce al 35,2\% cuando la sustentadora es una mujer) y menos en exclusión moderada. En resumen, los hogares encabezados por mujeres tienden a situarse en posiciones intermedias, mientras que los encabezados por varones se encuentran más habitualmente en los extremos (tanto superiores como inferiores) del continuum integración-exclusión.
Gráfico 6. Distribución de hogares con algún miembro de origen extracomunitario o de la ampliación de la UE en el espacio de la integración/exclusión, según el sexo de la persona sustentadora principal. España, 2013 (\%)

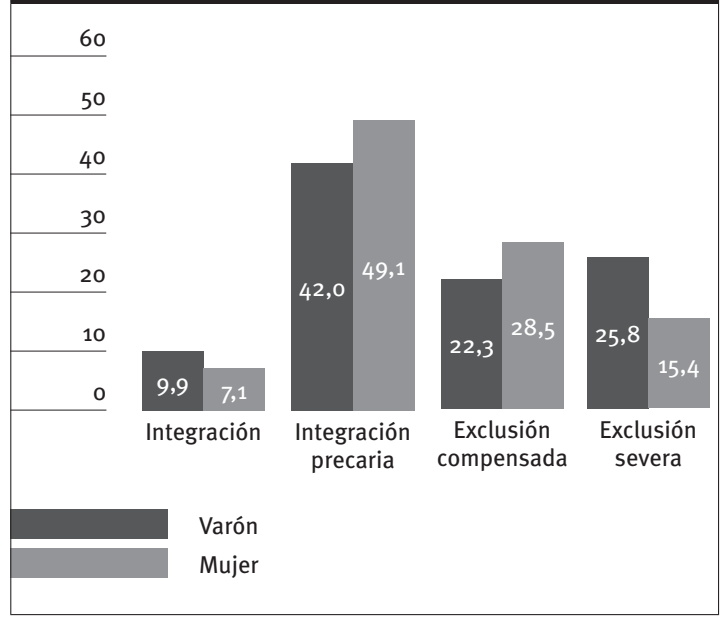

Fuente: Elaboración propia a partir de la Encuesta Foessa 2013.

Si observamos la evolución de la exclusión a partir de sus distintas dimensiones, la fotografía que obtenemos es distinta, y la ventaja femenina antes descrita se reduce claramente. En este caso, de hecho, los hogares encabezados por mujeres siguen posicionándose peor que los encabezados por hombres en muchos aspectos. Más en detalle, experimentan un mayor riesgo de conflicto social (12,7\% frente a 5,9\%) y niveles más elevados de exclusión de la vivienda (51,9\% frente a $46,9 \%)^{7}$, el empleo (61,4 \% frente a $58,2 \%)$, el consumo (13,5\% frente a $12,6 \%$ ) y la educación (7,5\% frente a $6,9 \%)$. Se posicionan mejor sólo en el ámbito político (el riesgo de exclusión en esta dimensión es del 56,2 \% para los hogares encabezados por una mujer, frente al $60,6 \%$ de los encabezados por un hombre) y de la salud ( $27,8 \%$ frente a $32 \%)$. ¿Cómo es posible que, en 2013 , los hogares con sustentadora principal mujer presenten, por un lado, un menor riesgo de exclusión globalmente considerado y un ISES más reducido y, por otro, mayores niveles de exclusión en las distintas dimensiones? La respuesta está en razones de orden metodológico: la exclusión en una dimensión concreta no implica necesariamente que también haya exclusión en general. Es decir, que un hogar puede presentar exclusión en una esfera, pero situarse bien en todas las demás, con lo cual no se considerará que es un hogar excluido. En suma, los datos obtenidos nos dicen que los hogares cuyo sueldo principal es aportado por una mujer, aun presentando menores niveles de exclusión que los hogares con sustentador principal varón, siguen, sin embargo, enfrentando dificultades en un gran número

7 Destacamos que el peor posicionamiento respecto a la vivienda se deriva prácticamente de un solo indicador (gastos excesivos en vivienda), mientras que en otros seis son los hogares encabezados por hombres los que obtienen peores resultados. Estos datos sugieren que, en este caso, la exclusión de la vivienda remite más a la dimensión económica que a la de vivienda propiamente dicha. Es decir, este indicador probablemente refleja los peores sueldos de las mujeres en comparación con los de los hombres. 
de esferas (y, por ello, se sitúan más fácilmente que los hogares encabezados por hombres en la zona de integración precaria, y menos en la de integración plena).

Tabla 3. Distribución de los hogares con algún miembro de origen extracomunitario o de la ampliación de la UE que experimentan exclusión, en sus distintas dimensiones, según el sexo de la persona sustentadora principal. España, 2007 y 2013 (\%)

\begin{tabular}{|l|c|c|c|c|}
\cline { 2 - 5 } \multicolumn{1}{c|}{} & \multicolumn{2}{c|}{2007} & \multicolumn{2}{c|}{2013} \\
\cline { 2 - 5 } \multicolumn{1}{c|}{} & Varón & Mujer & Varón & Mujer \\
\hline Exclusión del empleo & 28,3 & 29,9 & 58,2 & 61,4 \\
\hline Exclusión del consumo & - & - & 12,6 & 13,5 \\
\hline Exclusión política & 75,8 & 95,9 & 60,6 & 56,2 \\
\hline Exclusión de la educación & 5,1 & 7,2 & 6,9 & 7,5 \\
\hline Exclusión de la vivienda & 29,6 & 51,5 & 46,9 & 51,9 \\
\hline Exclusión de la salud & 19,1 & 18,4 & 32,0 & 27,8 \\
\hline Conflicto social & 7,3 & 15,5 & 5,9 & 12,7 \\
\hline Aislamiento social & 2,5 & 29,9 & 5,6 & 3,0 \\
\hline
\end{tabular}

Fuente: Elaboración propia a partir de las Encuestas Foessa 2007 y 2013.

\subsection{Hogares con sustentador/a principal joven}

En relación a los hogares en los que la persona que aporta más ingresos tiene menos de 30 años, empezamos destacando que, para comprender su inclusión entre los hogares de riesgo, hay que tener en cuenta que, en 2013, la tasa de paro entre personas menores de 30 años era casi 20 puntos mayor que en el conjunto de la población ( $42,4 \%$ frente a $26,1 \%$, según la Encuesta de Población Activa).

Ante todo, observamos que, en el periodo considerado, la proporción de hogares con sustentador/a principal joven se ha reducido casi 3 puntos (del $9,5 \%$ en 2007 al $6,6 \%$ en 2013). En la base de esta reducción, se encuentran dos procesos paralelos: por un lado, el creciente número de jóvenes que se ven obligados a permanecer en el domicilio de los padres por la imposibilidad de costearse una vivienda propia; por otro, una creciente proporción de jóvenes ya independizados/as que, por las mismas razones, se ven obligados/as a retornar al domicilio de sus progenitores.

En segundo lugar, destacamos que, en el mismo periodo, la proporción de hogares cuyo sueldo principal es aportado por una mujer aumenta en casi 4 puntos (del 33,2\% en 2007 al 36,9\% en 2013). Nuevamente, recordamos el mayor impacto que la crisis parece haber tenido en sectores profesionales masculinizados. Por otra parte, destaca también que, tanto en 2007 como en 2013, la proporción de hogares en los cuales el sueldo principal es aportado por una mujer es mayor entre las y los jóvenes que el conjunto de la población. Este hecho puede reflejar procesos diferentes: en primer lugar, podría indicar una tendencia hacia la reducción de la división sexual del trabajo, lo cual reflejaría un cambio de calado estructural; en segundo lugar, podría indicar un mayor impacto de la crisis en el empleo masculinizado y joven, lo cual indicaría un cambio meramente coyuntural; finalmente, podría simplemente reflejar que, en edades tempranas, la participación de las mujeres en el mercado es mayor y en condiciones mejores que en cohortes más avanzadas, que coinciden con la llegada de hijos e hijas en el hogar. De todas formas, cabe destacar que las diferencias entre los hogares jóvenes y el conjunto de los hogares son reducidas, y tienden a disminuir (de 7 puntos en 2007 a 4 puntos en 2013) ${ }^{8}$.

Una vez aclaradas estas cuestiones de carácter preliminar, observamos cuáles son las condiciones de vida de estos hogares. La fotografía que obtenemos es muy interesante: si en 2007 las diferencias entre hogares encabezados por hombres y por mujeres eran tan significativas que estos últimos tenían el doble de probabilidades de vivir procesos de exclusión (15,8\% frente a 7,8\%), en 2013 la situación se invierte, y son los hogares encabezados por hombres los que viven mayores dificultades (32,1\% frente a $28,5 \%$ y un ISES un $5 \%$ más elevado). Es éste un cambio muy significativo y resaltarlo es importante; sin embargo, aquí también cabe destacar que la reducción de las desigualdades de género se ha obtenido a costa de un empeoramiento de la situación generalizado e intenso (el ISES, de hecho, se ha más que duplicado 2007 y 2013 , pasando de 0,83 a 1,72). Además, el hecho de que los hogares encabezados por mujeres estén mejor posicionados no indica que se haya logrado la igualdad de género, ya que incluso entre los hogares con sustentador/a principal joven, en la mayoría de los casos $(63,1 \%)$ la persona que aporta más ingresos es un hombre.

Gráfico 7. Distribución de los hogares con sustentador/a principal joven en situación de exclusión, según su sexo. España, 2007 y 2013 (\%)

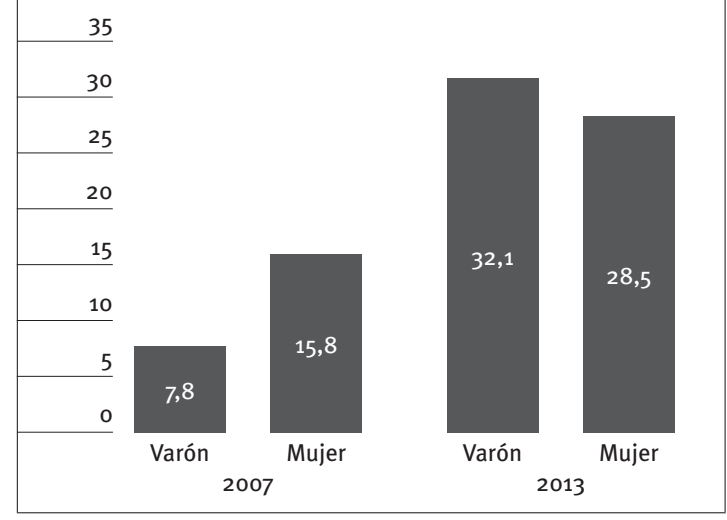

Fuente: Elaboración propia a partir de las Encuestas Foessa 2007 y 2013.

${ }^{8}$ Consideramos que esta reducción es puramente coyuntural y efecto del incremento, en el conjunto de la población, de los hogares encabezados por mujeres. 
Si afinamos el análisis y observamos cómo se distribuyen estos hogares en los cuatro espacios de integración/exclusión, observamos que, análogamente a cuanto sucedía en los hogares con algún miembro de origen extracomunitario o de la ampliación de la UE, los hogares encabezados por una mujer, aun presentando mayores probabilidades de integración, se sitúan más en integración precaria (39,9\% frente a $35,1 \%)$ y menos en integración plena $(31,5 \%$ frente a $32,9 \%)$. Por otra parte, si centramos la mirada en el espacio de la exclusión, observamos que, entre los hogares excluidos, aquellos que tienen sustentador principal varón presentan un mayor riesgo de encontrarse en exclusión severa (cuando el sustentador principal es un varón, el 43,6\% de los hogares excluidos se encuentran en exclusión severa, valor que se reduce al 29,3\% cuando es una mujer). En resumen, se confirma la tendencia, ya observada en el apartado anterior, de los hogares encabezados por mujeres a situarse más en posiciones intermedias, mientras que los hogares encabezados por varones se encuentran más habitualmente en los extremos (tanto superiores como inferiores) del continuum integración-exclusión.

Gráfico 8. Distribución de los hogares con sustentador/a principal joven en el espacio de la integración/exclusión, según su sexo. España, 2013 (\%)

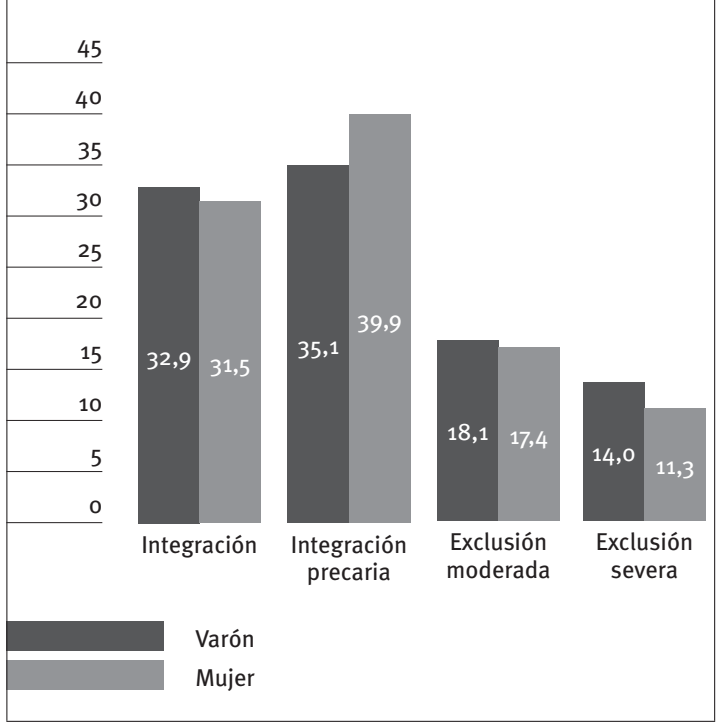

Fuente: Elaboración propia a partir de la Encuesta Foessa 2013.

Aquí también, al igual que en el caso de los hogares con algún miembro de origen extracomunitario o de la ampliación de la UE, si realizamos un análisis por dimensiones, descubrimos que los hogares encabezados por mujeres siguen, en muchos aspectos, posicionándose peor que los encabezados por hombres. De nuevo, las dimensiones en las que estos hogares enfrentan mayores dificultades son el conflicto social (13,6 \% frente a 4,4 \%), la vivienda $9(36,4 \%$

9 También en este caso, el peor posicionamiento se deriva solamente de dos indicadores (gastos excesivos y hacinamiento), mien- frente a $31 \%)$, el empleo (46,3\% frente a $43,6 \%)$, la educación (7,9\% frente a 5,2\%) y el consumo $(11,3 \%$ frente a $10,7 \%)$. Por el contrario, presentan menores niveles de exclusión sólo en la esfera política (19,2 \% frente a $24,4 \%$ ) y de la salud (12,2 \% frente a 15,4\%). Como ya se ha aclarado en el apartado anterior, estos resultados indican que los hogares cuyo sueldo principal es aportado por una mujer, aun presentando, en general, menores niveles de exclusión que los hogares con sustentador principal varón, siguen sin embargo enfrentando ingentes dificultades en un gran número de esferas.

Tabla 4. Distribución de los hogares con sustentador/a principal joven que experimentan exclusión, en sus distintas dimensiones, según su sexo. España, 2007 y 2013 (\%)

\begin{tabular}{|l|c|c|c|c|}
\cline { 2 - 5 } \multicolumn{1}{c|}{} & \multicolumn{2}{c|}{2007} & \multicolumn{2}{c|}{2013} \\
\cline { 2 - 5 } \multicolumn{1}{c|}{} & Varón & Mujer & Varón & Mujer \\
\hline Exclusión del empleo & 14,3 & 16,7 & 43,6 & 46,3 \\
\hline Exclusión del consumo & 1,3 & 1,8 & 10,7 & 11,3 \\
\hline Exclusión política & 31,7 & 21,9 & 24,4 & 19,2 \\
\hline Exclusión de la educación & 6,1 & 7,9 & 5,2 & 7,9 \\
\hline Exclusión de la vivienda & 13,9 & 12,3 & 31,0 & 36,4 \\
\hline Exclusión de la salud & 5,2 & 5,2 & 15,4 & 12,2 \\
\hline Conflicto social & 2,6 & 9,6 & 4,4 & 13,6 \\
\hline Aislamiento social & 6,1 & 7,0 & 4,1 & 2,8 \\
\hline
\end{tabular}

Fuente: Elaboración propia a partir de las Encuestas Foessa 2007 y 2013.

\subsection{Hogares monoparentales}

Para terminar nuestra presentación de los colectivos en situación de especial vulnerabilidad, focalizamos la mirada en los hogares monoparentales. Es éste un análisis especialmente relevante en el marco de los estudios de género de los procesos de exclusión, por dos razones. En primer lugar, porque se trata de hogares claramente sobrerrepresentados en el espacio de la exclusión, tal y como aclaran muchos autores y autoras (Gabàs, 2003; Jiménez, Morgado y Del Mar, 2004; Raya, 2004; Pérez y Laparra, 2007; Laparra y García, 2010; Laparra y Pérez, 2008). En segundo lugar, porque, con su rostro femenino y excluido, los hogares monoparentales ejemplifican claramente la existencia de profundas desigualdades de género que cruzan la sociedad.

Desde aquí consideramos que, para comprender la monoparentalidad, hay que tener en cuenta principalmente tres hechos: que se trata de un fenómeno en crecimiento; que da lugar a un tipo de hogar claramente feminizado; y que es un tipo de hogar que, en la actualidad, está claramente sobrerrepresentado en el espacio de la exclusión.

tras que en otros cinco son los hogares encabezados por hombres los que obtienen peores resultados. 
En primer lugar, por tanto, destacamos que, en los últimos seis años, el porcentaje de hogares monoparentales sobre el conjunto de hogares ha aumentado un $21,4 \%$ (del 9,8\% en 2007 al $10,3 \%$ en 2009 y al $11,9 \%$ en 2013). Este aumento, sin duda significativo, se muestra, sin embargo, aún más acentuado si, en lugar de limitar la mirada a los hogares monoparentales y mononucleares, la centramos en todos los hogares con algún núcleo monoparental en su interior $^{10}$. Entre 2007 y 2013, de hecho, la proporción de este tipo de hogares sobre el conjunto de hogares ha aumentado un $31,5 \%$ (del 10,8\% en 2007 al $11,7 \%$ en 2009 y al 14,2\% en 2013). Es más, si diferenciamos en función del sexo de la persona que aporta más ingresos, observamos que, cuando ésta es una mujer, el aumento es mucho más intenso que cuando es un varón ( $40 \%$ frente a $14 \%$ )

En segundo lugar, para comprender las dinámicas relacionadas con estos hogares, es necesario tener en cuenta que nos enfrentamos a un tipo de hogar claramente feminizado, tanto que algunas autoras hablan de 'monomarentalidad' en lugar que de monoparentalidad (Jiménez, Morgado y Del Mar 2004). Los datos, de hecho, revelan que, si en el conjunto de los hogares españoles sólo una pequeña minoría (33,2\%) recibe su sustento principal de una mujer, en el caso de los hogares monoparentales la situación se invierte, y en cuatro de cada cinco casos $(78,7 \%)$ la sustentadora principal es una mujer. En el caso de los hogares donde hay algún núcleo monoparental, la situación es parecida, aunque la feminización es algo menos intensa (71,9\% en 2013). Por otra parte, si consideramos que en el primer caso ésta se ha mantenido constante en los últimos años, mientras que en el segundo se ha incrementado (el $67,9 \%$ del total en 2007 al $71,9 \%$ en 2013 ), podemos suponer que las diferencias tienden a desaparecer.

Finalmente, si centramos nuestro análisis en los hogares en los que la sustentadora principal es una mujer ${ }^{11}$, observamos que mientras que en 2007 ni la monoparentalidad ni la presencia de núcleos monoparentales en el hogar representaban factores de exclusión (el ISES, de hecho, se situaba en o,96 en el primer caso y en o,98 en el segundo), la situación cambia radicalmente en 2009 y 2013. En este marco temporal, el porcentaje de hogares monoparentales en situación de exclusión aumenta tres veces y media, pasando del 11,2\% en 2007 al 30,7\% en 2009 $\mathrm{y}$ al $39,2 \%$ en 2013 . El ISES también da cuenta de este cambio y aumenta un 156\% (del 0,91 en 2007 al 2,33 en 2013). En el caso de los hogares con algún núcleo monoparental, la evolución es parecida, aunque el empeoramiento se presenta algo menos

${ }^{10}$ Mientras que los 'hogares monoparentales' son hogares mononucleares, compuestos solamente por una persona adulta y por las y los menores sobre los cuales ésta tiene la responsabilidad exclusiva, los 'hogares con algún núcleo parental' incluyen tanto hogares mononucleares como hogares bi- o polinucleares, siempre que uno de los núcleos que los conforma sea monoparental.

${ }^{11}$ Limitamos el análisis a los hogares encabezados por mujeres, porque en lo referente a los encabezados por hombres la muestra no es suficiente para garantizar la fiabilidad de los datos obtenidos. intenso: el riesgo de exclusión, de hecho, más que se triplica, pasando del 9,8\% en 2007 al 31,1\% en 2009 y al $33,7 \%$ en 2013 , y el ISES aumenta un $76,5 \%$ (del 0,98 en 2007 al 1,73 en 2013).

En ambos casos, se trata de un empeoramiento mucho más intenso que el que ha enfrentado el conjunto de los hogares: mientras que el riesgo de vivir procesos de exclusión aumentaba, entre 2007 y 2013, un 35,2 \% para los hogares encabezados por una mujer y un $39 \%$ para los encabezados por un varón, este incremento alcanzaba el $\mathbf{2 5 0} \%$ en el caso de los hogares monoparentales y superaba el $240 \%$ en el de los hogares con algún núcleo monoparental. En suma, si en 2007 estos hogares presentaban un riesgo de exclusión incluso menor que el resto, en 2013 la situación se invierte y el riesgo de exclusión se dispara. Para los hogares monoparentales, de hecho, el riesgo es ahora 15,4 puntos mayor en la generalidad de los hogares encabezados por una mujer y 18,2 puntos más elevado que entre los hogares donde el sueldo principal es aportado por un varón. En el caso de los hogares con algún núcleo monoparental, el panorama es parecido: 10 puntos mayor en el primer caso y casi 13 puntos más elevado en el segundo.

Como último punto, deseamos destacar que estos datos confirman plenamente una de las formulaciones del concepto de feminización de la pobreza presentadas al principio de este trabajo: la que nos habla de un aumento de la pobreza entre hogares encabezados por mujeres.

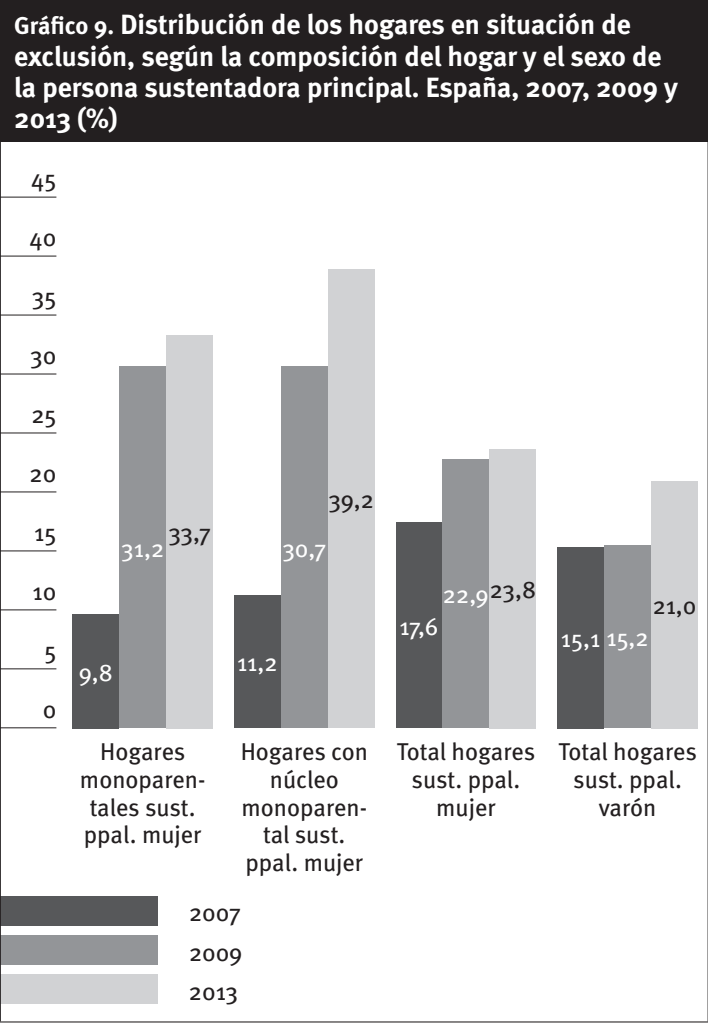

Fuente: Elaboración propia a partir de las Encuestas Foessa 2007, 2009 y 2013. 


\section{Conclusiones}

En este trabajo hemos realizado un estudio de cómo las desigualdades de género que cruzan la sociedad revierten también en los procesos de exclusión: por un lado, hemos presentado una fotografía de las desigualdades existentes en la actualidad; por otro, a este análisis estático se le ha acompañado de un estudio dinámico de las transformaciones que han tenido lugar entre 2007 y 2013. A partir de la información proporcionada por las Encuestas de la Fundación Foessa sobre Integración Social y Necesidades Sociales, hemos presentado datos tanto a nivel tanto individual como de hogar, tanto agregados como diferenciando las distintas dimensiones de la exclusión, y tanto relativos al conjunto de la población como focalizados en colectivos especialmente vulnerables.

Ante todo, es interesante destacar que los resultados son claramente distintos según realicemos un análisis por individuos o por hogares. En el primer caso, los datos nos dicen que, en los últimos siete años, las diferencias en el nivel de integración de hombres y mujeres han ido reduciéndose hasta prácticamente desaparecer. En el segundo caso, al contrario, aunque la distancia se ha acortado, permanece una clara desventaja de los hogares con sustentadora principal mujer.

Estas diferencias en función de la unidad de análisis se explican si consideramos que, en el primer caso, las desigualdades de género resultan parcialmente invisibilizadas e infrarrepresentadas: los datos de carácter individual presentados se han obtenido, de hecho, a partir de una información agregada relativa al hogar. Es decir, que a todos los individuos que componen un hogar se les ha atribuido automáticamente el mismo índice de exclusión social. Si partimos del presupuesto de que la mayoría de los hogares se componen tanto de mujeres como de hombres, se comprende que esta forma de medir el nivel de exclusión, aunque justificada por el hecho de que los hogares constituyen unidades de consumo, de redistribución interna de recursos, de creación de solidaridades y apoyos (Laparra 2014), puede resultar engañosa a la hora de medir las desigualdades de género. Por ello, un acercamiento a las desigualdades existentes entre hombres y mujeres requiere también de un análisis a nivel de hogares.

Pese a estas diferencias, un dato se muestra con toda claridad, independientemente de la unidad de análisis considerada: la reducción de las desigualdades de género en los últimos seis años. Una mirada superficial podría inducirnos a celebrar acríticamente esta tendencia. Un análisis más meditado, sin embargo, nos obliga a matizar nuestras conclusiones, ya que su interpretación es ambivalente. Por un lado, estos datos podrían estar reflejando una tendencia estable hacia la reducción de las desigualdades de género, es decir, una transformación de carácter estructural; por otro, sin embargo, podría muy bien tratarse de algo puramente coyuntural, reflejo de una crisis que ha impactado más en sectores profesionales masculinizados. Esta última hipótesis es claramente negativa, sobre todo porque la constatación de una menor desigualdad entre géneros podría llegar a debilitar las políticas de igualdad, cuando en realidad su necesidad seguiría invariada.

Independientemente del significado que se atribuya a estas tendencias, dos hechos están claros: por un lado, la reducción de las desigualdades de género en los últimos años; por otro, la persistencia de cierta desventaja de los hogares encabezados por mujeres. Si ponemos estos datos en relación con las distintas formulaciones del concepto de feminización de la pobreza presentadas al principio de este trabajo, podemos concluir que mientras que se confirma la hipótesis de una sobrerrepresentación de las mujeres en la franja de pobreza (exclusión), se desmiente la idea de un aumento progresivo de la presencia de mujeres entre las personas pobres (excluidas).

Un análisis de género de los procesos de exclusión, sin embargo, no puede limitarse a estudiar la presencia o ausencia de exclusión y su intensidad. Al contrario, es necesario analizar cómo el género influye en las dinámicas que originan, alimentan y describen estos procesos. Para ello, se ha realizado un estudio por dimensiones de la exclusión: se ha podido así observar que, aunque las mujeres presentan mayores niveles de exclusión en todas las dimensiones, las diferencias son más elevadas en la esfera socialrelacional ( 9,7 puntos), se reducen ligeramente en el eje político (donde la distancia entre hogares encabezados por hombres y por mujeres se sitúa en 7,1 puntos) y son claramente menores en la esfera económica (donde no superan los 3 puntos). Estos datos sugieren la existencia de importantes diferencias en los factores que están en la base de los procesos de exclusión de mujeres y hombres. Se vería, por lo tanto, confirmada la acepción del concepto de feminización de la pobreza que defiende que las mujeres se empobrecen por razones y procesos específicos y condicionados por el género.

Por otra parte, es también necesario destacar que el análisis aquí realizado no es ciertamente exhaustivo: para comprender cómo el género incide tanto en los factores que desencadenan tales procesos como en su intensidad y los mecanismos que se utilizan para salir de ellos (Pérez y Laparra, 2007), es necesario, de hecho, disponer de otro tipo de datos: nos referimos a datos longitudinales, que permitan observar cómo ha evolucionado la situación de un individuo/hogar a lo largo del tiempo (Laparra y Aguilar, 2000); 0, en caso de que eso no sea posible, a información de carácter cualitativo, que permita analizar itinerarios y visibilizar tendencias (Pérez y Laparra, 2007). Resumiendo, sin embargo, podemos afirmar que, aunque los datos de los que disponemos no nos ofrecen una imagen detallada de cómo el género influye en los factores desencadenantes de la exclusión, sí nos permiten afirmar con toda seguridad que existen diferencias en función de esta variable.

Con respecto a la dimensión económica, hemos obtenido dos resultados interesantes. Por un lado, 
los datos revelan que cuando los dos miembros de una pareja tienen un empleo, el riesgo de exclusión y pobreza es mucho menor que cuando sólo se emplea uno de ellos. Por otro, el análisis realizado también revela que, en el supuesto de que sólo uno de ellos tenga empleo, el riesgo es mucho mayor cuando la persona empleada es la mujer y menor cuando es el varón. Estos datos nos llevan a afirmar con fuerza, por un lado, la necesidad de impulsar la incorporación laboral femenina y, por otro, la importancia de mejorar la calidad del empleo de las mujeres. Esto significa que las políticas de conciliación deberán reforzarse, pero, sobre todo, repensarse para favorecer su utilización por parte de los varones y evitar así que la conciliación se convierta en un 'asunto de mujeres', como de facto ha venido siendo hasta ahora. En concreto, es necesario encontrar un camino distinto del que han seguido los países del centro y norte de Europa (Suecia y Noruega, pero también los Países Bajos, Alemania o el Reino Unido), donde las políticas existentes no han logrado eliminar las desigualdades entre mujeres y hombres en lo que respecta al mercado de trabajo, sino sólo desplazarlas. Si bien es verdad que las tasas de ocupación femenina allí son mayores, también lo es que esto se ha logrado a costa de incrementar-para ellas, pero no para ellos - las cuotas de empleos a tiempo parcial (según datos Eurostat), con todo lo que esto implica en términos de sueldo o posibilidad de ascenso.

Por otra parte, para mejorar nuestro conocimiento de cómo el género incide en los procesos de exclusión no es suficiente analizar qué está sucediendo en general, sino que es necesario observar también cuál ha sido la evolución en algunos colectivos especialmente vulnerables. Más en detalle, nos referimos a los hogares con algún miembro de origen extracomunitario o de la ampliación de la UE, a los hogares con sustentador/a principal joven, y a los hogares monoparentales.

En el primer y segundo casos, las tendencias observadas para el conjunto de los hogares se ven elevadas a la enésima potencia: mientras que, en general, la ventaja masculina se reduce, pero no desaparece, en el caso de los hogares con alguna persona de origen extranjero o menor de 30 años, sí lo hace. Es decir, que se pasa de una ventaja masculina extremadamente intensa en 2007 a cierta ventaja femenina en 2013. Por otra parte, también hay que decir que, aunque en 2013 los hogares encabezados por mujeres estén ligeramente sobrerrepresentados en el espacio de la integración, siguen infrarrepresentados en el de la integración plena, donde se mantiene una clara ventaja masculina.

En el caso de los hogares monoparentales, se destaca que, en los últimos seis años, el riesgo de exclusión ha aumentado más intensamente que en cualquier otro colectivo: se pasa de un ISES del 0,98 en 2007 (es decir, ligeramente menor que para el conjunto de los hogares) a uno del 1,73 en 2013 (es decir, más del 30 \% más elevado que para la generalidad de los hogares). Por lo que respecta al concepto de feminización de la pobreza, estos datos claramente respaldan la formulación que defiende que la pobreza en hogares encabezados por mujeres está aumentado.

Como último punto, queremos terminar el presente trabajo realizando algunas apreciaciones acerca del concepto de feminización de la pobreza. En concreto, destacamos que los datos empíricos confirman algunas de las acepciones, mientras que contradicen otras. Esta ambivalencia nos lleva a sugerir que se abandone el concepto de feminización de la pobreza a favor de otros, como feminización de la exclusión, análisis de género de la exclusión social o sobrerrepresentación femenina en el espacio de la exclusión. Se trata de conceptos de alguna manera relacionados, pero no coincidentes. El primero es una clara evolución de la noción de feminización de la pobreza $\mathrm{y}$, por lo tanto, trae consigo tanto su fuerza como parte de su indeterminación y debe ser abordado con cautela. Es más, desde aquí consideramos que la noción de feminización identifica un proceso y, por lo tanto, que el uso de este concepto es idóneo sólo cuando el riesgo de exclusión femenino ha aumentado en un horizonte temporal dado (Medeiros y Costa, 2008; Kim y Choi, 2013). La noción de sobrerrepresentación femenina en el espacio de la exclusión, al contrario, no identifica un proceso, sino un estado, y, por lo tanto, puede ser utilizada con referencia a una realidad estática. Finalmente, el análisis de género de la exclusión social pone el acento en el hecho de que, a la hora de investigar, se ha tenido como objetivo primario visibilizar la incidencia del género en los procesos de exclusión, En nuestro trabajo, por tanto, podemos afirmar que se ha realizado un análisis de género de la exclusión social que, en término generales, ha evidenciado la existencia de una sobrerrepresentación femenina en el espacio de la exclusión, pero no de una feminización de la exclusión, fenómeno que ha afectado sólo al caso específico de los hogares monoparentales. 
AGUILAR, P. L. (2011): “La feminización de la pobreza: conceptualizaciones actuales y potencialidades analíticas”, Revista Katálysis, vol. 14, ํㅜ 1, págs. 126-133 [<http://dx.doi.org/10.1590/ S1414-49802011000100014)].

BRADY, D.; y KALL, D. (2008): “Nearly universal, but somewhat distinct: The feminization of poverty in affluent Western democracies, 1969-2000", Social Science Research, vol. 37, nํ3, págs. 976-1.007.

BRUNET , I.; VALLS , F.; y BELZUNEGUI , Á. (2008): “Poverty, social exclusion and gender”, Sistema, nำ 207, págs. $69-85$.

CABRERA CABRERA, P. J. (2004): "Pobreza y exclusión desde la perspectiva de género", en GARCÍA-MINA, A.; y CARRASCO GALÁN, Ma J. (eds.), Género y desigualdad: 'la feminización de la pobreza', Madrid, Universidad Pontificia Comillas, págs. 11-53.

DAMONTI, P. (2014): Una mirada de género a la exclusión social, serie Documento de Trabajo del VII Informe sobre Exclusión y Desarrollo Social en España, no 3.1, Madrid, Fundación Foessa; Cáritas Española Editores [<http://www. foessa2014.es/informe/uploaded/documentos_ trabajo/15102014151359_2687.pdf〉].

DE ELEJABEITIA, C. (1996): "Feminización de la pobreza", Documentación Social, no105, págs. 171-182.

EUROSTAT (2014): Persons Employed Part Time [rhttp:// ec.europa.eu/eurostat/tgm/table.do?tab=tab le $\&$ init $=1 \&$ language $=$ en $\&$ pode $=$ tpso0159\&pl ugin=1>].

FERNÁNDEZ VIGUERA, B. (1998): “Feminización de la pobreza en Europa y procesos de exclusión social”, en HERNÁNDEZ ARISTU, J.; y OLZA, $M$. (comps.), La exclusión social. Reflexión y acción desde el trabajo social, Madrid, Eunate, págs. 315-335.

- (1992): "Pobreza, marginación y exclusión. Feminización de la pobreza", en VV.AA., La sociedad de la desigualdad: pobreza y marginación a debate, Donostia, Tercera Prensa, págs. 77-86.

GABÀS I GASA, A. (2003): Indicadores de género contra la exclusión social. Informe sobre el fenómeno de la exclusión social, Surt-Asociació de Dones per a Inserció Laboral.

GAVIRIA, M.; LAPARRA, M.; y AGUILAR, M. (1995): "Aproximación teórica al concepto de exclusión”, en VV.AA., Desigualdad y pobreza hoy, Madrid, Talasa, págs. 133-200.

IZQUIERDO BENITO, M. J. (2010): "Las dos caras de la desigualdad entre mujeres y hombres: explotación económica y libidinal”, Quaderns de Psicología, vol. 12, no 2, págs. 117-129.

JIMÉNEZ, I.; MORGADO, B.; y DEL MAR GONZÁLEZ M. (2004): "Familias monomarentales y exclusión social", Portularia, vol. 4, págs. 249-259.

KIM, J. W.; y CHOI, Y. J. (2013): “Feminisation of poverty in 12 welfare states: Consolidating cross-regime variations?", International Journal of Social Welfare, vol. 22, no 4, págs. 347-359.

LAPARRA, M. (coord.) (2014): "La fractura social se ensancha: intensificación de los procesos de exclusión en España durante 7 años", en VII Informe sobre exclusión y desarrollo social en España, Madrid, Fundación Foessa; Cáritas Española Editores, págs. 151-255 [<http://www. foessa2014.es/informe/uploaded/descargas/ VII_INFORME.pdf)].

- (coord.) (2013): The Human Face of the Crisis in Spain. Report for the European Commission, Comision 
Europea, Dirección General de Empleo, Asuntos Sociales e Inclusión.

(2010): “El impacto de la crisis en la cohesión social o el surf de los hogares españoles en el modelo de integración de la 'sociedad líquida”', Documentación Social, nº 158, págs. 97-130.

LAPARRA, M.; y GARCÍA, A. (2010): “Alteraciones en el espacio social de la exclusión en un contexto de crisis económica", en LAPARRA, M.; y PÉREZ ERANSUS, B. (coords.), El primer impacto de la crisis en la cohesión social en España, Madrid, Fundación Foessa, págs. 59-92.

LAPARRA, M.; y PÉREZ ERÁNSUS, B. (2008): “¿Qué entendemos por exclusión social?”, en RENES, V. (coord.), VI Informe Foessa. Informe sobre exclusión social y desarrollo social en España, Madrid, Fundación Foessa, págs. 177-206.

LAPARRA, M.; N. ZUGASTI; y GARCÍA LAUTRE, I. (2014): Reflexiones metodológicas en el análisis de la exclusión social, serie Documento de Trabajo del VII Informe sobre Exclusión y Desarrollo Social en España, nํㅜ 3.11, Madrid, Fundación Foessa; Cáritas Española Editores [<http://www. foessa2014.es/informe/uploaded/documentos trabajo/28112014004407_3133.pdf>].

LAPARRA, M. et al. (2007): "Una propuesta de consenso sobre el concepto de exclusión. Implicaciones metodológicas", Revista Española del Tercer Sector, $\mathrm{n}-5$, págs. $15-57$.

MATEO PÉREZ, M. A. (2000): “Dos perspectivas metodológicas para la inclusión de la perspectiva de género en el análisis de la pobreza”, Psicohtema, vol. 12, no 2, págs. 377-381.

MEDEIROS, M.; y COSTA, J. (2008): "Is there a feminization of poverty in Latin America?", World Development, vol. 36, n- 1, págs. 115-127.

PAUGAM, S. (2001): "Les formes contemporaines de la pauvreté et de l'exclusion en Europe", Études Rurales, $\mathrm{n}$-159-160, págs. 73-96.
PÉREZ ERÁNSUS, B.; y LAPARRA, M. (2007): “Agenda de investigación para profundizar en el conocimiento de la exclusión social en España", en VIDAL FERNÁNDEZ, F.; y RENES AYALA, V., La agenda de investigación en exclusión y desarrollo social, Madrid, Fundación Foessa; Cáritas Española Editores, págs. 131-148.

RAYA DÍEZ, E. (2004): “Exclusión social y ciudadanía: claroscuros de un concepto", Aposta. Revista de Ciencias Sociales, ํㅜㅇ, págs. 1-18.

RODRÍGUEZ CABRERO, G. (2004): “Los límites del Estado del bienestar. La transición hacia la nueva reforma social”, en RODRÍGUEZ CABRERO, G., El Estado del bienestar en España: debates, desarrollo y retos, Madrid, Fundamentos, págs. 15-44.

SCOT, H. (1984): Working Your Way to the Bottom: The Feminization of Poverty, Londres, Pandora Press.

SUBIRATS, J. (dir.) (2004): Pobreza y exclusión social: un análisis de la realidad española y europea, Barcelona, Fundación La Caixa.

TORTOSA, J. M. (2009): "Feminización de la pobreza y perspectiva de género", Revista Internacional de Organizaciones, $\mathrm{n}-3$, págs. 71-89.

TRUJILLO CARMONA, M. (2014): Diseño muestral de la Encuesta sobre Integración Social y Necesidades Sociales, serie Documento de Trabajo del VII Informe sobre Exclusión y Desarrollo Social en España, no 3.10, Madrid, Fundación Foessa; Cáritas Española Editores [<http://www.foessa2014. es/informe/uploaded/documentos trabajo/23102014144958_7141.pdf>].

UGT NAVARRA (2014): Las brechas de género rompen la tendencia a la igualdad en el mercado laboral en el último año, UGT Navarra. 